\title{
High-dimensional characterization of post-acute sequelae of COVID-19
}

https://doi.org/10.1038/s41586-021-03553-9

Received: 18 January 2021

Accepted: 14 April 2021

Published online: 22 April 2021

Check for updates

\author{
Ziyad Al-Aly ${ }^{1,2,3,4,5 \bowtie}$, Yan Xie ${ }^{1,2,6}$ \& Benjamin Bowe $e^{1,2,6}$
}

The acute clinical manifestations of COVID-19 have been well characterized ${ }^{1,2}$, but the post-acute sequelae of this disease have not been comprehensively described. Here we use the national healthcare databases of the US Department of Veterans Affairs to systematically and comprehensively identify 6 -month incident sequelae-including diagnoses, medication use and laboratory abnormalities-in patients with COVID-19 who survived for at least 30 days after diagnosis. We show that beyond the first 30 days of illness, people with COVID-19 exhibit a higher risk of death and use of health resources. Our high-dimensional approach identifies incident sequelae in the respiratory system, as well as several other sequelae that include nervous system and neurocognitive disorders, mental health disorders, metabolic disorders, cardiovascular disorders, gastrointestinal disorders, malaise, fatigue, musculoskeletal pain and anaemia. We show increased incident use of several therapeutic agents-including pain medications (opioids and non-opioids) as well as antidepressant, anxiolytic, antihypertensive and oral hypoglycaemic agents-as well as evidence of laboratory abnormalities in several organ systems. Our analysis of an array of prespecified outcomes reveals a risk gradient that increases according to the severity of the acute COVID-19 infection (that is, whether patients were not hospitalized, hospitalized or admitted to intensive care). Our findings show that a substantial burden of health loss that spans pulmonary and several extrapulmonary organ systems is experienced by patients who survive after the acute phase of COVID-19. These results will help to inform health system planning and the development of multidisciplinary care strategies to reduce chronic health loss among individuals with COVID-19.
COVID-19 is a viral illness caused by the coronavirus SARS-CoV-2. The acute clinical manifestations of COVID-19 have been well characterized and involve both pulmonary and extrapulmonary systemic manifestations ${ }^{1,2}$. Emerging reports suggest that-for some individuals-the symptoms of COVID-19 persist beyond the acute setting. However, the post-acute sequelae of COVID-19 are not yet clear.

Here we leveraged the breadth and depth of the US Department of Veterans Affairs electronic health databases to undertake a highdimensional approach to comprehensively identify the 6-month outcomes of incident diagnoses (from 379 diagnostic categories), incident medication use (from 380 medication classes) and incident laboratory abnormalities (from 62 laboratory tests) in people who survived for at least the first 30 days after their COVID-19 diagnosis.

\section{Non-hospitalized patients with COVID-19}

The cohort included 73,435 users of the Veterans Health Administration (VHA) with COVID-19 who survived for at least the first 30 days after their COVID-19 diagnosis and who were not hospitalized, and 4,990,835 VHA users who did not have COVID-19 and were not hospitalized (Supplementary Fig. 1a, b). The median follow-ups were 126 (81-203; for all reported median values, parenthetical ranges refer to the interquartile range) and 130 (82-205) days for patients with COVID-19 and VHA users, respectively (Extended Data Table 1a). We examined a panel of negative-outcome controls, which yielded results that were consistent with our a priori expectations (for example, hazard ratios of 1.03 (0.94-1.12; for all hazard ratios and burdens, parenthetical ranges refer to $95 \%$ confidence intervals) and 1.03 (0.95-1.12) for neoplasms and accidental injuries, respectively); the results of all the negative-outcome controls are provided in Extended Data Table 2a. Our examination of the standardized differences of all high-dimensional variables across all outcome-specific cohorts (including those that were selected and those that were not selected in the models) showed that more than $99.99 \%$ of standardized differences were $<0.1$ after adjustment (Supplementary Fig. 2a, b), which resulted in similar distributions of baseline characteristics in each group after adjustment (Supplementary Table 1).

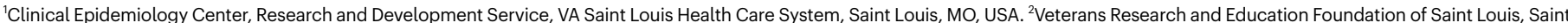

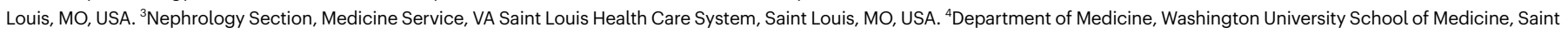
Louis, MO, USA. Institute for Public Health, Washington University in Saint Louis, Saint Louis, MO, USA. ${ }^{6}$ Department of Epidemiology and Biostatistics, College for Public Health and Social Justice, Saint Louis University, Saint Louis, MO, USA. ${ }^{凶}$ e-mail: zalaly@gmail.com 
Beyond the first 30 days of illness, individuals with COVID-19 had an increased risk of death (hazard ratio of 1.59 (1.46-1.73)). We also estimated the adjusted excess burden of death due to COVID-19 per 1,000 persons at 6 months on the basis of the difference between the estimated incidence rate in individuals with COVID-19 and all VHA users. The excess death was estimated at 8.39 (7.09-9.58) per 1,000 patients with COVID-19 at 6 months. Individuals with COVID-19 had a higher risk of requiring outpatient care (hazard ratio of $1.20(1.19-1.21)$ ), at an excess burden of 33.22 (30.89-35.58; all excess burdens are given per 1,000 patients with COVID-19 at 6 months) and at a greater frequency of $0.47(0.44-0.49)$ additional encounters every 30 days (Extended Data Table 2b, c).

We evaluated the risk of incident occurrence of 379 diagnoses (that were categorized according to ICD-10 codes based on Clinical Classifications Software Refined), 380 classes of medication and 62 laboratory tests beyond the first 30 days. For each of the outcomes we examined, we built a cohort who were free of the related outcome at baseline to identify the risk of incident outcome during follow-up. We found that several conditions in almost every organ system exhibited an adjusted hazard ratio that was greater than 1 and a $P$ value lower than $6.57 \times 10^{-5}$ (significance level adjusted for multiple comparisons). The adjusted hazard ratio and burden for all outcomes are presented in Fig. 1a-c and Supplementary Tables $2-4$. The result for outcomes that were positively associated with COVID-19 are presented in Fig. 2a-c, Extended Data Fig. 1a-c, Supplementary Table 5 and are discussed here.

\section{Respiratory conditions}

The most common excess burden at 6 months after a COVID-19 infection that did not result in a hospitalization in the first 30 days was that of respiratory conditions, which included respiratory signs and symptoms (excess burden of $28.51(26.40-30.50)$ ), respiratory failure, insufficiency and arrest (3.37 (2.71-3.92)), and lower respiratory disease (4.67 (3.96-5.28)). There was also evidence of a high burden of incident use of bronchodilators (22.23 (20.68-23.67)), antitussive and expectorant agents (12.83 (11.61-13.95)), anti-asthmatic agents (8.87 (7.65-9.97)) and glucocorticoids (7.65 (5.67-9.50)).

\section{Diseases of the nervous system}

An excess burden of nervous system conditions was also evident, and included nervous system signs and symptoms (14.32 (12.16-16.36)), neurocognitive disorders (3.17 (2.24-3.98)), nervous system disorders (4.85 (3.65-5.93)) and headache (4.10 (2.49-5.58)).

\section{Mental health burden}

Our results also showed an excess burden of sleep-wake disorders (14.53 (11.53-17.36), anxiety and fear-related disorders (5.42 (3.427.29)), and trauma- and stress-related disorders (8.93 (6.62-11.09)). These findings were coupled with evidence of excess burden of incident use of non-opioid (19.97 (17.41-22.40)) and opioid (9.39 (7.21-11.43)) analgesic drugs, antidepressant agents (7.83 (5.19-10.30)), and benzodiazepine, sedative and anxiolytic agents (22.23 (20.68-23.67)).

\section{Metabolic disorders}

An excess burden of several metabolic disorders was evident, including disorders of lipid metabolism (12.32(8.18-16.24)), diabetes mellitus (8.23 $(6.36,9.95))$ and obesity $(9.53(7.55-11.37))$. These was also evidence of an excess burden of incident use of antilipaemic agents (11.56 (8.73-14.19)), oral hypoglycaemic drugs (5.39 (3.99-6.64)) and insulin (4.95(3.87-5.90)), as well as an excess burden of elevated low-density lipoprotein cholesterol (9.48 (7.02-11.81)), total cholesterol $(9.94(6.61,13.11))$, triglycerides $(9.40$ (6.63-12.03)) and haemoglobin A1c (10.66 (6.77-14.35)).

\section{Poor general wellbeing}

Individuals with COVID-19 exhibited an excess burden of poor general wellbeing, including malaise and fatigue (12.64 (11.24-13.93)), muscle disorders (5.73 (4.60-6.74)), musculoskeletal pain (13.89 (9.89-17.71)) and anaemia (4.79 (3.53-5.93)). These diagnoses were coupled with laboratory evidence of an excess burden of anaemia, comprising decreased haemoglobin (31.03 (28.16-33.76)), decreased haematocrit levels $(30.73(27.64,33.67))$ and low serum albumin $(6.44(4.84,7.92))$.

\section{Cardiovascular conditions}

There was an excess burden of cardiovascular conditions, including hypertension (15.18 (11.53-18.62)), cardiac dysrhythmias (8.41 (7.18$9.53))$, circulatory signs and symptoms $(6.65(5.18-8.01))$, chest pain (10.08 (8.63-11.42)), coronary atherosclerosis (4.38 (2.96-5.67)) and heart failure (3.94 (2.97-4.80)). There was also evidence of excess burden of incident use of beta blockers (9.74 (8.06-11.27)), calcium channel blockers (7.18 (5.61-8.61)), loop diuretic agents (4.72 (3.59-5.72)), thiazide diuretic agents (2.52 (1.37-3.54)), and anti-arrhythmic drugs (1.28 (0.79-1.67)).

\section{Gastrointestinal system}

There was evidence of an excess burden of the following conditions: oesophageal disorders (6.90 (4.58-9.07)), gastrointestinal disorders (3.58 (2.15-4.88)), dysphagia (2.83 (1.79-3.76)) and abdominal pain (5.73 (3.7-7.62)). These conditions were coupled with evidence for an increased use of laxatives $(9.22(6.99-11.31))$, anti-emetic agents $(9.22$ (6.99-11.31)), histamine antagonists $(4.83(3.63-5.91))$, other antacids (1.07 (0.62-1.42)) and antidiarrhoeal agents (2.87 (1.70-3.91)). Laboratory abnormalities included an increased risk of incident high levels of alanine aminotransferase (7.62 (5.20-9.90)).

\section{Other sequelae}

There was also evidence of an excess burden in incident acute pulmonary embolism (2.63 (2.25-2.92)) and use of anticoagulant drugs (16.43 (14.85-17.89)). Other conditions included excess burden of skin disorders (7.52 (5.17-9.73)), arthralgia and arthritis (5.16 (3.18-7.01)) and infections, including urinary tract infections (2.99 (1.94-3.93)) (Fig. 2a-c, Supplementary Tables 2-5).

\section{COVID-19 requiring hospitalization versus influenza}

To gain a better understanding of the spectrum of clinical manifestations in patients with COVID-19 who were hospitalized, we undertook a comparative evaluation of a cohort of hospitalized individuals with COVID-19 versus individuals who were hospitalized with seasonal influenza (a well-known and well-characterized respiratory viral illness).

This cohort included 13,654 people with COVID-19 and 13,997 people with influenza who survived for at least 30 days after hospital admission (Supplementary Fig. 3a, b). The median follow-ups were 150 (84-217) and 157 (87-220) days for patients with COVID-19 and influenza, respectively (Extended Data Table 1a). We tested a panel of negative-outcome controls, which yielded results that were consistent with our a priori expectations (for example, hazard ratio of $0.98(0.83-1.16)$ and 1.02 (0.90-1.15) for neoplasms and accidental injuries, respectively); the results of all the negative-outcome controls are provided in Extended Data Table 2a. Our examination of standardized differences of all high-dimensional variables (including those that were selected and those that were not selected in the models) in all outcome-specific cohorts showed that more than $99.75 \%$ of standardized differences were $<0.1$ after adjustment (Supplementary Fig. $4 a, b$ ), which resulted 

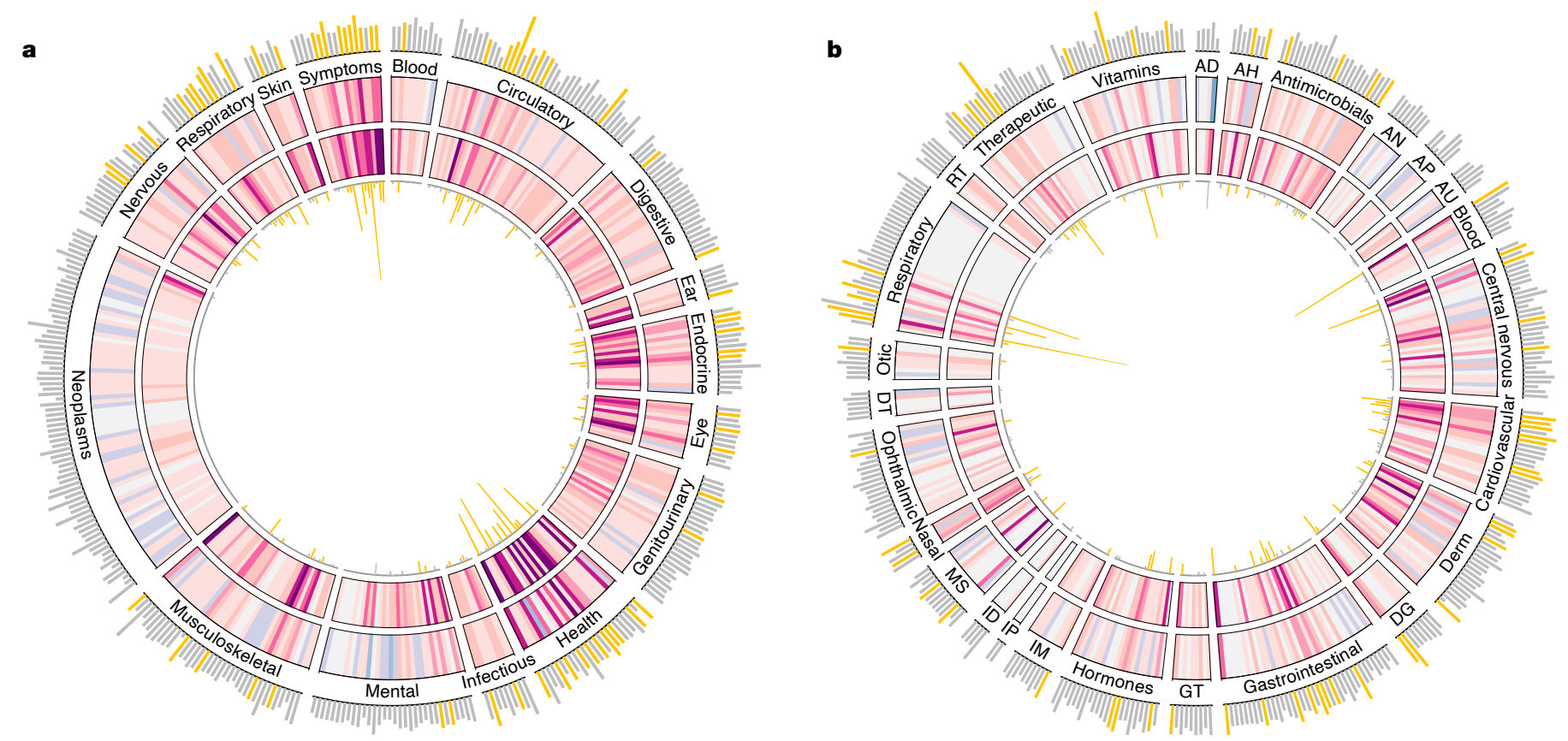

c

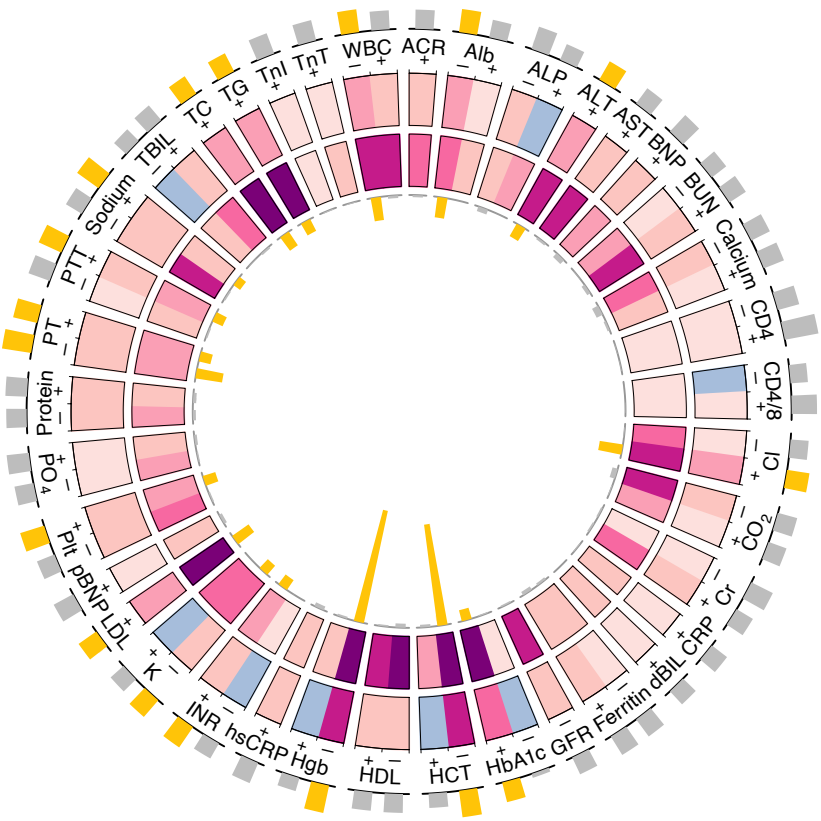

Fig. 1 High-dimensional identification of the incident post-acute sequelae of COVID-19. a-c, Incident diagnoses (a), incident medication use (b) and incident laboratory abnormalities (c). All VHA users served as the referent category. Post-acute sequelae were ascertained from 30 days after infection until end of follow-up. Beginning from the outside ring, the first ring represents hazard ratios for the post-acute sequelae of COVID-19. A higher bar indicates a larger hazard ratio. Hazard ratios with a point estimate larger than one and that was statistically significant are shown in yellow. The second ring represents the excess burden per 1,000 patients with COVID-19 at 6 months. The colour of the cell indicates the value of the excess burden (deeper shades of red indicate a higher excess burden and deeper shades of blue indicate a greater reduced burden). The third ring represents the baseline incident rate in the control group (deeper shades of red indicate a higher incident rate). The fourth ring represents negative log of the $P$ value; a higher bar indicates a smaller $P$ value and yellow indicates that the value is statistically significant. ACR, albumin/

creatinine ratio; $\mathrm{AD}$, antidotes; $\mathrm{AH}$, antihistamine drugs; Alb, albumin; $\mathrm{ALP}$, alkaline phosphatase; ALT, alanine aminotransferase; AN, antineoplastic agents; AP, antiparasitic agents; AST, aspartate aminotransferase; AU, autonomic; BNP, brain natriuretic peptide; BUN, blood urea nitrogen; CD4, CD4 cell count; $C D 4 / 8, C D 4 / C D 8$ ratio; $C l$, chloride; $C r$, creatinine; $C R P$, C-reactive protein; dBIL, direct bilirubin; Derm, dermatological; DG, diagnostic; DT, dental; GFR, glomerular filtration rate; GT, genitourinary; HbA1c, haemoglobin A1c; HCT, haematocrit; HDL, high-density-lipoprotein cholesterol; Hgb, haemoglobin; hsCRP, high-sensitivity C-reactive protein; ID, irrigation or dialysis; IM, immunological; INR, international normalized ratio; IP, intrapleural; K, potassium; LDL, low-density-lipoprotein cholesterol; MS, musculoskeletal; pBNP, pro-B natriuretic peptide; PIt, platelet; Protein, total protein; $\mathrm{PT}$, prothrombin time; $\mathrm{PTT}$, partial thromboplastin time; $\mathrm{RT}$, rectal; TBIL, total bilirubin; TC, total cholesterol; TG, triglycerides; TnI, troponin I; $\mathrm{TnT}$, troponin $\mathrm{T} ; \mathrm{WBC}$, white blood cell. 


\begin{tabular}{|l|l|}
\hline Respiratory signs and symptoms & $28.51(26.40-30.50)$ \\
\hline Hypertension & $15.18(11.53-18.62)$ \\
\hline Sleep-wake disorders & $14.53(11.53-17.36)$ \\
\hline Nervous system signs and symptoms & $14.32(12.16-16.36)$ \\
\hline Musculoskeletal pain (not low back pain) & $13.89(9.89-17.71)$ \\
\hline Malaise and fatigue & $12.64(11.24-13.93)$ \\
\hline Disorders of lipid metabolism & $12.32(8.18-16.24)$ \\
\hline Chest pain & $10.08(8.63-11.42)$ \\
\hline Obesity & $9.53(7.55-11.37)$ \\
\hline Trauma- and stressor-related disorders & $8.93(6.62-11.09)$ \\
\hline Cardiac dysrhythmias & $8.41(7.18-9.53)$ \\
\hline Diabetes mellitus & $8.23(6.36-9.95)$ \\
\hline Skin disorders (itch, rash or other) & $7.52(5.17-9.73)$ \\
\hline Oesophageal disorders & $6.90(4.58-9.07)$ \\
\hline Circulatory signs and symptoms & $6.65(5.18-8.01)$ \\
\hline Abdominal pain & $5.73(3.70-7.62)$ \\
\hline Muscle disorders & $5.73(4.60-6.74)$ \\
\hline Anxiety- and fear-related disorders & $5.42(3.42-7.29)$ \\
\hline Arthralgia and arthritis & $5.16(3.18-7.01)$ \\
\hline Nervous system disorders & $4.85(3.65-5.93)$ \\
\hline Anaemia & $4.79(3.53-5.93)$ \\
\hline Lower respiratory disease & $4.67(3.96-5.28)$ \\
\hline Chronic obstructive pulmonary disease & $4.44(3.16-5.59)$ \\
\hline Genitourinary signs and symptoms & $4.39(2.98-5.68)$ \\
\hline Coronary atherosclerosis and other heart disease & $4.38(2.96-5.67)$ \\
\hline Headache & $4.10(2.49-5.58)$ \\
\hline Heart failure & $3.94(2.97-4.80)$ \\
\hline Gastrointestinal disorders & $3.58(2.15-4.88)$ \\
\hline Respiratory failure, insufficiency or arrest & $3.37(2.71-3.92)$ \\
\hline Neurocognitive disorders & $3.17(2.24-3.98)$ \\
\hline Acute phlebitis, thrombophlebitis or thromboembolism & $3.05(2.51-3.49)$ \\
\hline Urinary tract infections & $2.99(1.94-3.93)$ \\
\hline Dysphagia & $2.83(1.79-3.76)$ \\
\hline Asthma & $2.82(1.92-3.61)$ \\
\hline Acute pulmonary embolism & $2.63(2.25-2.92)$ \\
\hline Bacterial infections & $2.38(1.52-3.13)$ \\
\hline Pressure ulcer of skin & $2.05(1.40-2.59)$ \\
\hline Pleurisy or pleural effusion & $1.52(0.95-1.98)$ \\
\hline & \\
\hline
\end{tabular}

b

\begin{tabular}{|l|l|}
\hline Bronchodilators (sympathomimetic or inhalation) & $22.23(20.68-23.67)$ \\
\hline Non-opioid analgesics & $19.97(17.41-22.40)$ \\
\hline Anticoagulants & $16.43(14.85-17.89)$ \\
\hline Non-opioid-containing antitussives or expectorants & $12.83(11.61-13.95)$ \\
\hline Antilipaemic agents & $11.56(8.73-14.19)$ \\
\hline NSAIDs & $10.94(8.04-13.67)$ \\
\hline Beta blockers & $9.74(8.06-11.27)$ \\
\hline Topical anti-inflammatories & $9.63(6.74-12.37)$ \\
\hline Opioid analgesics & $9.39(7.21-11.43)$ \\
\hline Laxatives & $9.22(6.99-11.31)$ \\
\hline Antiasthmatics & $8.87(7.65-9.97)$ \\
\hline Antidepressants & $7.83(5.19-10.30)$ \\
\hline Vitamin D & $7.80(5.36-10.09)$ \\
\hline Glucocorticoids & $7.65(5.67-9.50)$ \\
\hline Vitamin C & $7.23(6.45-7.90)$ \\
\hline Calcium channel blockers & $7.18(5.61-8.61)$ \\
\hline Nasal anti-inflammatories & $6.33(4.57-7.96)$ \\
\hline Anticonvulsants & $5.78(3.68-7.72)$ \\
\hline Oral hypoglycaemic agents & $5.39(3.99-6.64)$ \\
\hline Topical antifungals & $5.10(3.37-6.69)$ \\
\hline Insulin & $4.95(3.87-5.90)$ \\
\hline Zinc & $4.90(4.39-5.32)$ \\
\hline Penicillins & $4.87(3.15-6.44)$ \\
\hline Histamine antagonists & $4.83(3.63-5.91)$ \\
\hline Skeletal muscle relaxants & $4.78(2.62-6.79)$ \\
\hline Loop diuretics & $4.72(3.59-5.72)$ \\
\hline Topical nasal and throat agents & $4.13(3.09-5.05)$ \\
\hline Potassium & $3.72(2.35-4.96)$ \\
\hline Iron & $3.57(2.46-4.56)$ \\
\hline Magnesium & $3.36(2.27-4.32)$ \\
\hline Antiemetics & $3.07(1.66-4.36)$ \\
\hline Cyanocobalamin & $2.98(1.69-4.14)$ \\
\hline Antidiarrheal agents & $2.87(1.70-3.91)$ \\
\hline Thiazides or related diuretics & $2.52(1.37-3.54)$ \\
\hline Vaccines & $2.43(1.43-3.31)$ \\
\hline Multivitamins & $2.31(1.40-3.10)$ \\
\hline Anti-inflammatories (inhalation) & $1.37(0.80-1.83)$ \\
\hline Antiarrhythmics & $1.28(0.79-1.67)$ \\
\hline Magnesium-containing antacids & $1.07(0.62-1.42)$ \\
\hline & \\
\hline
\end{tabular}

Haemoglobin lower than 14 (M) or $12(\mathrm{~F}) \mathrm{g} \mathrm{dl}^{-1}$ Haematocrit lower than $42 \%(M)$ or $37 \%(F)$ Haemoglobin A1C higher than $5.6 \%$ Triglycerides higher than $150 \mathrm{mg} \mathrm{dl}^{-1}$ $\mathrm{dl}^{-1}-9.48(7.02-11.81)$ \begin{tabular}{l|l} 
Total cholestel high $200 \mathrm{mg} \mathrm{dl}^{-1}$ & $9.40(6.63-12.03)$
\end{tabular} Serumchloide higher $9.21(7.05-11.24)$ Total white blood cell count lower than 4,800 per $\mathrm{mm}^{3} \quad 8.45(6.47-10.29)$ $\begin{array}{ll}\text { Alanine aminotransferase higher than } 40 \mathrm{U} \mathrm{I}^{-1} & 7.62(5.20-9.90)\end{array}$ Serum albumin lower than $3.5 \mathrm{~g} \mathrm{dl}^{-1} \quad 6.44$ (4.84-7.92) Prothrombin time lower than $11.5 \mathrm{~s} \quad 4.81(3.88-5.64)$ \begin{tabular}{l|l} 
Serum potassium lower than $3.5 \mathrm{mmol} \mathrm{I}^{-1}$ & $4.44(2.92-5.85)$
\end{tabular} Platelet count higher than 400,000 per $\mathrm{mm}^{3} \quad 3.05(2.10-3.88)$ \begin{tabular}{ll} 
Platelet count higher than 400,000 per mm $^{3}$ & $3.05(2.10-3.88)$ \\
\hline Prothrombin time higher than $14.7 \mathrm{~s}$ & $2.99(2.00-3.86)$ \\
\hline
\end{tabular} \begin{tabular}{ll}
\hline Prothrombin time higher than $14.7 \mathrm{~s}$ & $2.99(2.00-3.86)$ \\
\hline International normalized ratio higher than 1.2 ratio & $2.94(1.96-3.80)$ \\
\hline
\end{tabular} \begin{tabular}{ll} 
Partial thromboplastin time higher than $36.5 \mathrm{~s}$ & $2.66(1.75-3.46)$ \\
\hline
\end{tabular} \begin{tabular}{l|l} 
Serum sodium higher than $145 \mathrm{mmol}^{-1}$ & $1.64(0.97-2.20)$
\end{tabular}

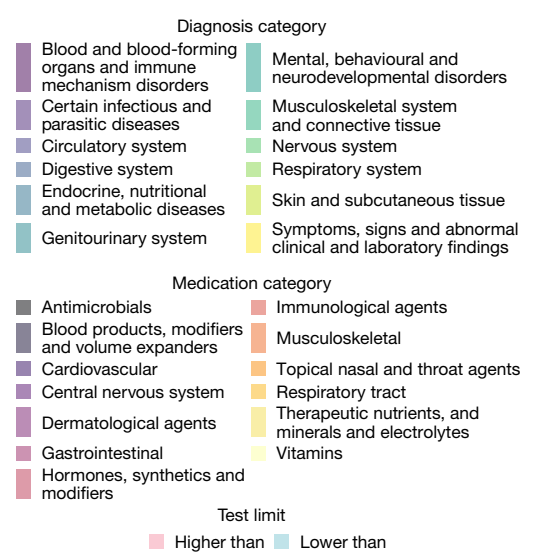

are presented with $95 \%$ confidence intervals in parentheses. Outcomes are ranked within each domain on the basis of the excess burden, from high to low. Diagnoses are coloured on the basis of the diagnosis group, medications are coloured on the basis of their class and laboratory abnormalities are coloured on the basis of their being higher or lower than the normal range. $F$, female; $\mathrm{M}$, male; NSAIDs, non-steroidal anti-inflammatory drugs. in similar distributions of baseline characteristics in each group after adjustment (Supplementary Table 6).

Beyond the first 30 days of illness, individuals with COVID-19 who had been hospitalized for this disease had an increased risk of death (hazard ratio of 1.51 (1.30-1.76)); we estimated excess death at 28.79 (19.52-36.85) per 1,000 persons at 6 months. Individuals with COVID-19 exhibited a higher risk of requiring outpatient care (hazard ratio of 1.12 (1.08-1.17)), at an excess burden of 6.37 (4.01-9.03) and with greater frequency of $1.45(1.28-1.63)$ additional encounters every 30 days (Extended Data Table 2b, c).

Compared to individuals who were hospitalized with seasonal influenza (and beyond the first 30 days of illness), patients who had been hospitalized for COVID-19 had a higher burden of a broad array of pulmonary and extrapulmonary systemic manifestations, including neurological disorders (burdens of 19.78 (12.58-26.19) and 16.16 (10.40-21.19) for nervous system disorders and neurocognitive disorders, respectively), mental health disorders (for example, a burden of 7.75 (4.72-10.10) for mental and substance-use conditions), metabolic disorders (for example, a burden of 43.53 (28.71-57.08) for disorders of lipid metabolism), cardiovascular disorders (for example, a burden of $17.92(10.73-24.35)$ for circulatory signs and symptoms), gastrointestinal disorders (for example, a burden of 19.28 (12.75-25.13) for dysphagia), coagulation disorders (14.31(10.08-17.89)), pulmonary embolism (18.31 (15.83-20.25)) and other disorders including malaise and fatigue (36.49 (28.13-44.15)) and anaemia (19.08 (10.58-26.81)) (Extended Data Figs. 2a-f, 3a-c, Supplementary Tables 7-10). Analyses of risk and the burden of clinical manifestations that additionally adjusted for the severity of the acute infection yielded consistent results in both the direction and magnitude of estimates (Extended Data Figs. 4a-f, 5a-c, Supplementary Tables 11-14). Our high-dimensional comparative evaluation of six-month outcomes in a cohort of hospitalized individuals with COVID-19 $(n=13,654)$ versus individuals who were hospitalized for other causes $(n=901,516)$ yielded consistent results (Extended Data Figs. 6a-f, 7a-c, Supplementary Tables 15-18).

\section{Analysing risk of prespecified COVID-19 outcomes}

To complement our high-dimensional approach and to gain a deeper understanding of the clinical manifestations of post-acute COVID-19 across the severity of the initial acute disease, we evaluated the risks of a panel of prespecified outcomes across the care setting of the acute phase of the disease (using whether individuals were non-hospitalized, hospitalized or admitted to intensive care, as a proxy indicator of disease severity) and benchmarked risk in these populations to a common reference group (the broader population of the Veterans Affairs Health Care System $(n=4,990,835)$ ) (Extended Data Table 1b). Our assessment of standardized differences across the four groups showed that none of these differences was less than 0.1 after adjustment (Supplementary Fig. 5). Our results reveal (1) an increased risk of a broad array of specific clinical manifestations that include acute coronary disease, arrythmias, acute kidney injury, chronic kidney disease, memory problems and thromboembolic disease (Fig. 3, Supplementary Tables 19, 20); (2) that this risk was evident even in individuals who were not hospitalized with COVID-19; and (3) a risk gradient that increased across the care setting of the acute COVID-19 infection from non-hospitalized individuals to those who were hospitalized, and risk was highest in patients who were admitted to intensive care (Fig. 3, Supplementary Tables 19, 20).

To gain a better understanding of whether these post-acute, prespecified outcomes are unique to COVID-19 or whether they represent a general post-viral syndrome, we further conducted comparative analyses 


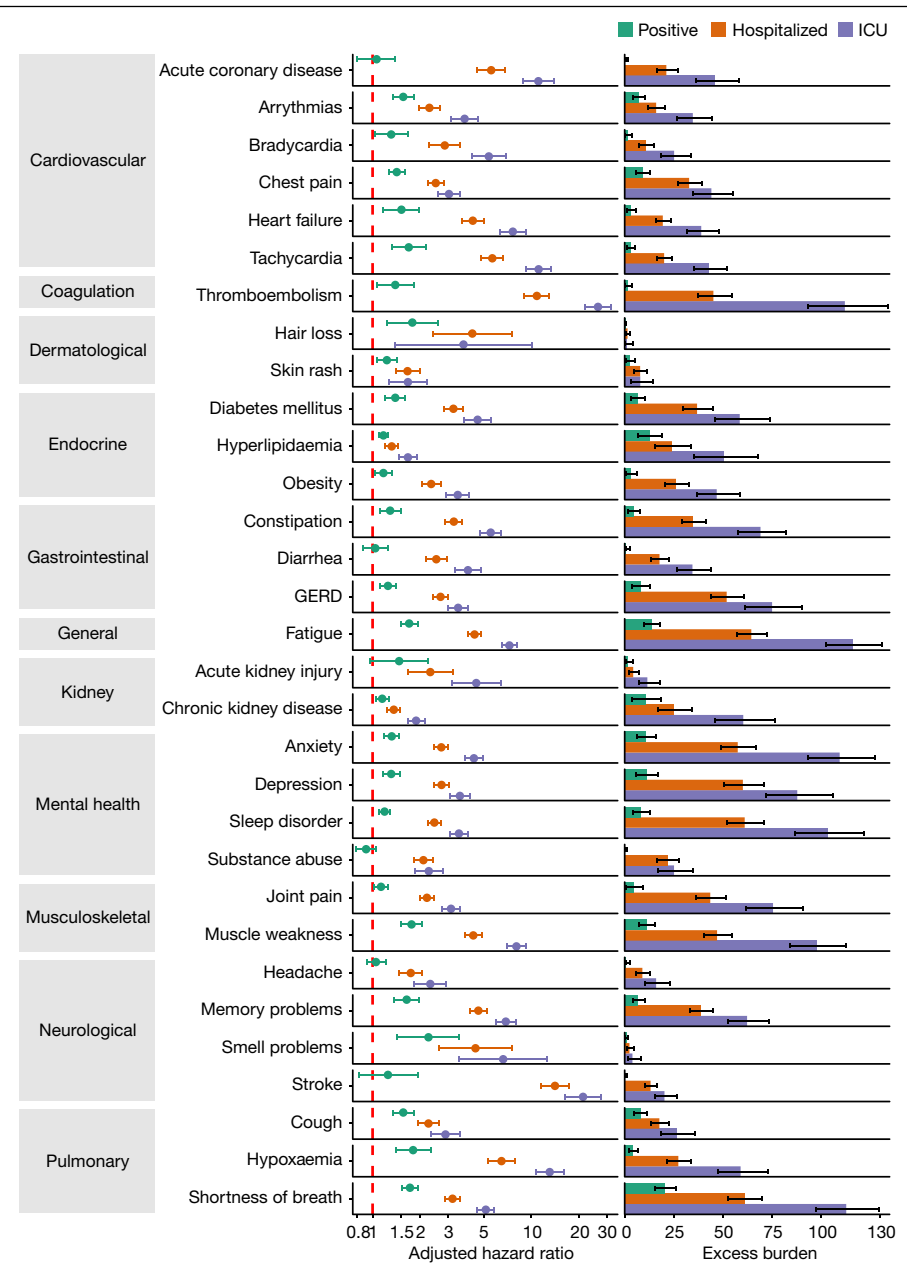

Fig. 3 | Risks and burdens of incident prespecified high-resolution post-acute COVID-19 outcomes. Risks and burdens were assessed at 6 months in mutually exclusive cohorts comprising non-hospitalized individuals with COVID-19, people who were hospitalized for COVID-19 and people who were admitted to intensive care for COVID-19 during the acute phase (first 30 days) of the infection. All VHA users served as the referent category. Outcomes were ascertained from day 30 after COVID-19 diagnosis until the end of follow-up. Adjusted hazard ratios and excess burdens are presented; error bars represent the $95 \%$ confidence interval. GERD, gastrointestinal reflux disease; ICU, intensive care unit.

(which were adjusted as specified in Methods, including adjusting for the severity of the acute infection) of the prespecified outcomes among people who were hospitalized with COVID-19 or seasonal influenza (Extended Data Table 1a, Supplementary Table 6). Our results show an increased risk and excess burden of a broad array of symptoms as well as multiple organ involvement among people with COVID-19 (Extended Data Fig. 8, Supplementary Table 21).

\section{Negative-exposure controls}

In addition to testing negative-outcome controls (Extended data Table 2a) and to further test the robustness of our approach, we developed and tested a pair of negative-exposure controls. We posited that exposure to influenza vaccination in odd-and even-numbered months between 1 October 2017 and 30 September 2019 should be associated with similar risks of clinical outcomes. We therefore tested associations between exposure to influenza vaccine in even- $(n=762,039)$ versus odd- $(n=599,981)$ numbered months and the full complement of 821 high-dimensional clinical outcomes considered in this study (including all diagnoses, medications and laboratory test results).
We used the same data sources, cohort-building algorithm, variable definitions, analytical approach (including weighting method) and outcome specification, as well as a similar length of follow-up and interpretation method. Our results showed that none of the associations met the threshold of significance $\left(P<6.57 \times 10^{-5}\right)$ considered in this study (Supplementary Fig. 6, Supplementary Tables 22-24).

\section{Discussion}

Here we use a high-dimensional approach to identify the spectrum of clinical abnormalities (incident diagnoses, incident medication use and incident laboratory abnormalities) experienced by individuals with COVID-19 who survive beyond the first 30 days of illness. The results suggest that, beyond the first 30 days of illness, people with COVID-19 are at higher risk of death and are more likely to use healthcare resources, and exhibit a broad array of incident pulmonary and extrapulmonary clinical manifestations (including nervous system and neurocognitive disorders, mental health disorders, metabolic disorders, cardiovascular disorders and gastrointestinal disorders) as well as signs and symptoms related to poor general wellbeing (including malaise, fatigue, musculoskeletal pain and anaemia). We observed an increased risk of the incident use of several classes of medication, including pain medications (opioid and non-opioid), antidepressant, anxiolytic, antihypertensive, antihyperlipidaemic and oral hypoglycaemic drugs and insulin. Our analyses of prespecified outcomes complement the high-dimensional approach to identify specific post-acute sequelae with greater diagnostic resolution and reveal two key findings: (1) that the risk and associated burden of post-acute sequelae is evident even among individuals whose acute disease was not severe enough to require hospitalization (representing the majority of people with COVID-19) and (2) that the risk and associated burden increases across the severity spectrum of the acute COVID-19 infection (from non-hospitalized to hospitalized individuals, to those admitted to intensive care). Our comparative approach to examining post-acute sequelae in individuals who are hospitalized with COVID-19 versus individuals with seasonal influenza (using a high-dimensional approach and through examination of prespecified outcomes) suggests that there is a substantially higher burden of a broad array of post-acute sequelae in the individuals who are hospitalized with COVID-19, which provides features that differentiate post-acute COVID-19 (both in the magnitude of risk and the breadth of organ involvement) from a post-influenza viral syndrome. Our results show that individuals who survive for 30 days or more after their COVID-19 diagnosis exhibit an increased risk of death and are more likely to use health resources, as well as a substantial burden of health loss that spans the pulmonary and several extrapulmonary organ systems; this highlights the need for holistic and integrated multidisciplinary long-term care of patients with COVID-19.

The mechanism or mechanisms that underlie the post-acute manifestations of COVID-19 are not entirely clear. Some of the manifestations may be driven by a direct effect of the viral infection, and may be explained by virus persisting in immune-privileged sites, an aberrant immune response, hyperactivation of the immune system or autoimmunity ${ }^{3}$. Indirect effects-including changes in social (for example, reduced social contact and loneliness), economic (for example, loss of employment) and behavioural conditions (for example, changes in diet and exercise) - that may be differentially experienced by people with COVID-19 may also shape health outcomes, and may be drivers of some of the post-acute clinical manifestations ${ }^{4-8}$. A better delineation of the direct and indirect effects, and a deeper understanding of the underlying biological mechanisms and epidemiological drivers, of the multifaceted long-term consequences of COVID-19 is needed ${ }^{9}$.

To our knowledge, this is the largest study of the post-acute sequelae of COVID-19; it involves 73,435 non-hospitalized patients with COVID-19, and 4,990,835 control individuals (corresponding to 2,070,615.52 person years of follow-up), as well as 13,654 hospitalized patients with 


\section{Article}

COVID-19 and 13,997 patients hospitalized with seasonal influenza (corresponding to 12,179.05 person years of follow-up). We leveraged the breadth and depth of the national healthcare databases of the US Department of Veterans Affairs (the largest nationally integrated healthcare delivery system in the US) to undertake a comprehensive high-dimensional comparative approach (relative to control groups) to identify the 6-month health outcomes and clinical manifestations in patients who survived the first 30 days of COVID-19. We further examined risk in a prespecified set of outcomes with higher diagnostic resolution across care settings to enable a deeper understanding of the clinical symptomatology and diagnoses of post-acute COVID-19 across the spectrum of severity of the acute phase of the infection.

This study has several limitations. Although our approach identifies the incident post-acute sequelae in patients with COVID-19, it does not delineate which sequelae may be direct or indirect consequences of COVID-19 infection. Because of the predominantly male composition of the Veterans Affairs population, our findings may not identify clinical features of post-acute COVID-19 that may be much more pronounced in women, or non-expressed or very rare in men. Our approach demonstrated balance for more than 1,150 variables across several data domains (diagnoses, medications and laboratory data) and yielded successful testing of negative-exposure and -outcome controls, but we cannot completely rule out residual confounding effects. Finally, as the global pandemic of COVID-19 continues to evolve, as treatment strategies improve, as new variants of the virus emerge and as vaccine availability increases, it is likely that the epidemiology and short- and long-term outcomes of COVID-19 will also change over time.

Our findings show that, beyond the first 30 days of illness, a substantial burden of health loss that spans pulmonary and several extrapulmonary organ systems is experienced by individuals who survived the acute phase of COVID-19. Our results will inform global discussions on the post-acute manifestations of COVID-19, as well as health system planning and the development of care strategies that are aimed at reducing chronic and permanent health loss and optimizing wellness among patients with COVID-19.

\section{Online content}

Any methods, additional references, Nature Research reporting summaries, source data, extended data, supplementary information, acknowledgements, peer review information; details of author contributions and competing interests; and statements of data and code availability are available at https://doi.org/10.1038/s41586-021-03553-9.

1. Wang, D. et al. Clinical characteristics of 138 hospitalized patients with 2019 novel coronavirus-infected pneumonia in Wuhan, China. J. Am. Med. Assoc. 323, 1061-1069 (2020)

2. Xie, Y., Bowe, B., Maddukuri, G. \& Al-Aly, Z. Comparative evaluation of clinical manifestations and risk of death in patients admitted to hospital with Covid-19 and seasonal influenza: cohort study. Br. Med. J. 371, m4677 (2020).

3. Advisory Group of the British Society for Immunology. Long-term Immunological Health Consequences of COVID-19 (British Society for Immunology, 2020).

4. Figueroa, J. D. et al. Distinguishing between direct and indirect consequences of Covid-19. Br. Med. J. 369, m2377 (2020)

5. Townsend, E. COVID-19 policies in the UK and consequences for mental health. Lancet Psychiatry 7, 1014-1015 (2020).

6. Knipe, D., Evans, H., Marchant, A., Gunnell, D. \& John, A. Mapping population menta health concerns related to COVID-19 and the consequences of physical distancing: a Google trends analysis. Wellcome Open Res. 5, 82 (2020).

7. Raker, E. J., Zacher, M. \& Lowe, S. R. Lessons from Hurricane Katrina for predicting the indirect health consequences of the COVID-19 pandemic. Proc. Natl Acad. Sci. USA 117, 12595-12597 (2020).

8. Mahase, E. Covid-19: mental health consequences of pandemic need urgent research, paper advises. Br. Med. J. 369, m1515 (2020)

9. Del Rio, C., Collins, L. F. \& Malani, P. Long-term health consequences of COVID-19. J. Am Med. Assoc. (2020)

Publisher's note Springer Nature remains neutral with regard to jurisdictional claims in published maps and institutional affiliations.

(C) This is a U.S. government work and not under copyright protection in the U.S.; foreign copyright protection may apply 2021 


\section{Methods}

All eligible participants were enrolled in the study, no statistical methods were used to predetermine sample size. The experiments were not randomized, and investigators were not blinded to allocation during experiments and outcome assessment.

\section{Setting}

Cohort participants were selected from US Department of Veterans Affairs (VA) electronic healthcare databases. The VHA provides healthcare to discharged veterans of the US armed forces and operates the largest nationally integrated healthcare system in the USA, with 1,255 healthcare facilities (including 170 VA Medical Centers and 1,074 outpatient sites) located across the USA. Veterans who are enrolled with the VHA have access to the comprehensive medical benefits package of the VA (which includes inpatient hospital care, outpatient services, preventive, primary and speciality care, prescriptions, mental healthcare, home healthcare, geriatric and extended care, medical equipment, and prosthetics). The VA electronic healthcare databases are updated daily.

\section{Cohort}

The cohort was constructed from 5,808,018 participants who had encountered the VHA between 1 January 2019 and 31 December 2019. Of those who were alive on 1 March $2020(n=5,606,309)$, a COVID-19 group was selected as individuals who had a positive test for COVID19 between 1 March 2020 and 30 November $2020(n=98,661)$. Participants without hospitalization within the first 30 days of their first positive test were further selected $(n=76,877)$. To examine post-acute outcomes, we then selected from the COVID-19 group those alive on the 30th day after their positive test (participants with COVID-19, $n=73,435$ ). To generate a comparison group that had a similar distribution of length of follow-up, we then matched each participant with COVID-19 with 70 VHA users who did not have a positive test for COVID-19 without replacement. In matching, the dates of cohort enrolment for the corresponding 70 VHA users were matched with time of cohort enrolment of the participant with COVID-19-that is, the date of testing positive (control group $n=5,140,450$ ). In the VHA user group, we similarly selected individuals who were without hospitalization and alive during the first 30 days after the date of enrolment (control group $n=4,990,835$ ) (Supplementary Fig. 1a, b). Participants were followed until 31 January 2021.

To compare post-acute outcomes of hospitalized participants with COVID-19 and hospitalized participants with seasonal influenza, we selected 15,846 participants with COVID-19 who were admitted to a hospital within 30 days after or 5 days before their first positive test (from the 98,661 patients with a positive COVID-19 test between 1 March 2020 and 30 November 2020). Similarly, we selected 62,909 patients who had their first positive seasonal influenza test between 1 October 2016 and 29 February 2020 and who had encountered the VHA at least once in the calendar year before the test was collected. Of these patients, 14,948 were admitted to a hospital within 30 days after or 5 days before their first positive influenza test. The hospitalized cohort was further restricted to those alive at the 30th day after hospital admission (COVID-19 $n=13,654$; seasonal influenza $n=14,212$ ), where for 215 patients who were in both the hospitalized COVID-19 and seasonal influenza group, only their COVID-19 hospitalizations were used in the analyses (Supplementary Fig. 3a, b). In this cohort, participants were considered to be enrolled at the time of hospitalization. To balance the duration of follow-up in the hospitalized COVID-19 and seasonal influenza groups, each participant in the seasonal influenza group was independently randomly assigned a duration of follow-up on the basis of the distribution of length of follow-up of the participants in the hospitalized COVID-19 group who were followed from date of hospitalization to 31 January 2021.
To examine high-resolution, prespecified post-acute COVID-19 outcomes across the severity spectrum of the initial acute disease, we built four mutually exclusive cohorts: VHA users without COVID-19 $(n=4,990,835)$, VHA users with COVID-19 $(n=73,435)$, VHA users who were hospitalized with COVID-19 within the first 30 days of follow-up $(n=10,068)$ and VHA users with COVID-19 who were admitted to the intensive care within the first 30 days of follow-up $(n=3,586)$. Participants in these cohorts were followed up until 31 January 2021.

\section{Data sources}

Electronic health records from VA Corporate Data Warehouse (CDW) were used in this study ${ }^{10-13}$. The CDW 'outpatient encounters' domains provided information related to outpatient encounters and 'inpatient encounters' domains provided information between hospital admission and discharge $\mathrm{e}^{14}$. The CDW 'outpatient pharmacy' domain and CDW 'bar code medication administration' domain were used to collect medication data, and CDW 'patient' domain was used to collect demographic information. The CDW 'laboratory results' domain was used to collect laboratory test information, and the 'COVID-19 shared data resource' was used to collect COVID-19 test and demographic information for patients with COVID-19. In addition, the area deprivation index-which is a composite measure of income, education, employment and housing-was obtained from the University of Wisconsin ${ }^{15}$.

\section{Post-acute use of health resources and death}

Outcomes that occurred after 30 days of cohort enrolment-including death, incident outpatient encounter and frequency of outpatient encounter-were examined in both cohorts. The frequency of outpatient encounters was computed on the basis of the number of days with outpatient encounter divided by days of follow-up after 30 days, and is reported as the number of outpatient encounters per 30 days.

\section{High dimensional post-acute clinical characteristics}

Negative outcome and exposure controls. The application of negative controls in clinical epidemiology may help to detect both suspected and unsuspected sources of spurious bias, and may lessen concerns about unmeasured confounding and other latent biases ${ }^{16}$. Here we followed a previously published approach ${ }^{16}$ to examine a panel of eight negative-outcome controls (including neoplasms, accidental injuries, scars, fitting or adjustment of orthodontic or dental prosthetic device, fitting or adjustment of hearing device, fitting or adjustment or orthotics, fitting or adjustment of casts, and bandages), for which (based on current knowledge) there should be no causal relation between the exposures and risks of the negative-outcome controls. We also developed and tested a pair of negative-exposure controls (defined as exposure to influenza vaccine in odd- or even-numbered months during the period between 1 October 2017 and 30 September 2019). We posited that there should be no differences in risk of clinical outcomes associated with receipt in influenza vaccine in odd-versus even-numbered months. The negative-exposure controls were tested in all 821 high-dimensional outcomes considered in our analyses, including diagnoses, medications and laboratory test results; we used the same data sources, cohort-building algorithm, variable definitions, analytical approaches and outcome specification, as well as a similar length of follow-up and interpretation method. In the assessment of negative-outcome and negative-exposure controls, the relation of the exposure-outcome pairs may share the same potential biases with COVID-19 and the outcomes examined in this study (including biases in the underlying data, algorithms for the construction of cohorts, unmeasured confounders, misspecification of modelling algorithms, outcome ascertainment, analytical considerations, result interpretation and other latent biases) ${ }^{16,17}$. The successful testing of negative controls reduces concerns about both suspected and unsuspected sources of spurious associations, including associations owing to unmeasured confounding, flaws in the analytical approach, differences 
in outcome ascertainment and other sources of bias ${ }^{16}$. In particular, the successful testing of the outcome controls may reduce concerns about biases in outcome ascertainment and unmeasured confounding between the comparison groups (for example, if there was bias in ascertainment of clinical outcomes in one arm versus another, this bias may also extend to ascertainment of neoplasms, accidental injuries or other negative-outcome controls tested in this study); the successful testing of the exposure control may reduce concerns about biases in the analytical approach and underlying data (for example, if there was bias related to the analytic approach, it may also bias the negative-exposure control).

Diagnoses. All ICD-10 diagnosis codes from cohort participants from day 30 after COVID-19 diagnosis until the end of follow-up were used to define the post-acute diagnosis outcomes. More than 70,000 ICD-10 diagnosis codes were classified into 540 diagnostic categories based on the Clinical Classifications Software Refined (CCSR) version 2021.1, which is developed as part of the Healthcare Cost and Utilization Project sponsored by the Agency for Healthcare Research and Quality ${ }^{18-20}$. We examined only diagnostic categories that may plausibly be considered post-acute sequelae of COVID-19 in the adult population. Some diagnostic categories-including external causes of morbidity, injury, poisoning and some other consequences of external causes, congenital malformations, deformations and chromosomal abnormalities, some conditions originating in the perinatal period or outcome from pregnancy, childbirth and the puerperium-were not examined, yielding 379 diagnostic categories.

Medication use. The prescription records of cohort participants from day 30 after COVID-19 diagnosis until the end of follow-up were used to define the post-acute medication use. We classified 3,425 medications on the basis of the VA drug classification system, into 543 medication classes $^{21,22}$. After removing items in the medication group of investigational agents or prosthetics, supplies and devices, we examined 380 different medication outcomes in total.

Laboratory abnormalities. In total, 62 laboratory test abnormalities from 38 laboratory measurements from day 30 after COVID-19 diagnosis until the end of follow-up were examined including absolute T cell count, alanine aminotransferase, aspartate aminotransferase, blood urea nitrogen, brain natriuretic peptide, C-reactive protein, carbon dioxide, CD4/CD8 ratio, direct bilirubin, estimated glomerular filtration rate, ferritin, haematocrit, haemoglobin, haemoglobin A1c, high-density-lipoprotein cholesterol, high-sensitivity C-reactive protein, international normalized ratio, low-density-lipoprotein cholesterol, microalbumin/creatinine ratio, partial thromboplastin time, platelet count, pro B natriuretic peptide, prothrombin time, serum albumin, serum alkaline phosphatase, serum calcium, serum chloride, serum creatinine, serum phosphate, serum potassium, serum sodium, serum total protein, total bilirubin, total cholesterol, total white blood cell count, triglycerides, troponin I and troponin $\mathrm{T}$ were identified on the basis of 'Logical Observation Identifiers Names and Codes'. Each laboratory test result was classified into abnormally high or abnormally low on the basis of whether results were above the upper normal range or below the lower normal range (in instances in which a high or low result might be clinically possible for a given laboratory test). The definition of the abnormality for each laboratory test is presented in Supplementary Tables 4, 9.

\section{High-resolution, prespecified post-acute COVID-19 outcomes}

To identify clinical manifestations of post-acute COVID-19 with greater diagnostic resolution, we specified a list of outcomes on the basis of data from the Center of Disease Control and the National Institute of Health workshop on post-acute COVID-19. Outcomes were defined on the basis of previous definitions that have been validated for use with electronic health records, and integrated information from diagnoses, medications and laboratory measurements when appropriate ${ }^{23-29}$. To gain a deeper understanding of the risks of these outcomes across the severity scale of the acute infection, we examined the risk across the care setting of the acute disease-a proxy indicator of clinical severity-in four mutually exclusive cohorts (VHA users (who served as the referent category); people with COVID-19; people hospitalized for COVID-19; and people admitted to intensive care for COVID-19). In addition, we estimated the risks of these prespecified outcomes in individuals hospitalized with COVID-19 and seasonal influenza. The prespecified, high-resolution outcomes included acute coronary disease, acute kidney injury, anxiety, arrythmias, bradycardia, chest pain, chronic kidney disease, constipation, cough, depression, diarrhoea, type 2 diabetes mellitus, fatigue, gastric oesophageal reflux disease, hair loss, headache, heart failure, hyperlipidaemia, hypoxaemia, joint pain, memory problems, muscle weakness, obesity, shortness of breath, skin rash, sleep disorder, smell disorder, stroke, tachycardia and thromboembolism. We restricted capture of incident acute coronary disease, stroke and thromboembolism to inpatient diagnoses that were not present on admission. All other prespecified outcomes that may plausibly be encountered in either the outpatient or inpatient setting were accordingly ascertained in the setting in which they first occurred. Among individuals with COVID-19, and for each prespecified outcome, the percentages of outcomes that were ascertained from outpatient and inpatient data are presented in Supplementary Tables 19, 20.

\section{Covariates}

The predefined covariates for analyses included demographics (such as age, race (white, black and other), sex and receipt of long-term care) and proxies of healthcare use (such as number of outpatient encounters, number of hospital admissions, number of outpatient prescriptions and number of outpatient eGFR measurements in the year before enrolment). In addition, we included the area deprivation index at the residency address of patients as a summary measurement of socio-economic deprivation. We used the Sequential Organ Failure Assessment (SOFA) score to adjust for the severity of the acute infection in additional high-dimensional analyses of the hospitalized COVID-19 versus hospitalized seasonal influenza cohorts ${ }^{30,31}$. To address potential nonlinear associations, all continuous variables were adjusted as restricted cubic spline functions.

To further adjust the models in the most optimal manner, we leveraged the multidimensionality of the electronic healthcare databases of the VA to algorithmically identify covariates (potential confounders) that span multiple domains (diagnoses, pharmacy records and laboratory tests) and that showed evidence of difference in prevalence between the comparison groups ${ }^{24}$. In the COVID-19 versus VHA users cohort (and separately in the hospitalized COVID-19 versus influenza cohort), high-dimensional covariates were ascertained within one year before the date of enrolment. Within all diagnoses, medication classes and laboratory tests, we first selected variables that occurs in at least 10 patients in both groups. We then estimated the unadjusted relative risk of each variable with being in the COVID-19 or comparator group. The top 100 high-dimensional variables with the strongest association with group membership were used, along with predefined covariates, in the analyses.

To most optimally estimate the risk of the set of prespecified outcomes across the intensity of care needed during the acute infection, we ascertained four sets of high-dimensional covariates (corresponding to the four mutually exclusive groups (all VHA users, people with COVID-19, people who were hospitalized with COVID-19 and people who were admitted to intensive care with COVID-19)) in total, on the basis of the unadjusted relative risk of being in each group compared to being in the remaining three groups. Highdimensional covariates were used along with predefined covariates in the analyses ${ }^{32}$. 


\section{Statistical analyses}

The characteristics of the VHA users who were not hospitalized for COVID-19, VHA users who were without COVID-19, hospitalized participants with COVID-19 and hospitalized participants with seasonal influenza are described in Extended Data Table 1a. The flow charts of the overall analytical approach are presented in Supplementary Figs. 7, 8.

We estimated the risk of health resource use and death, and the risk of each diagnosis, medication use and laboratory abnormality between individuals with COVID-19 and all VHA users, and-separately-between individuals who had been hospitalized for COVID-19 or seasonal influenza. To estimate the risk of each incident outcome, we built a cohort of participants without a history of the outcome being examined (for example, risk of insulin use was estimated within a cohort of participants without history of insulin use in the year before cohort enrolment). For each outcome-specific cohort, propensity scores based on predefined variables and high-dimensional algorithmically selected variables were estimated. The propensity scores were then used to compute the overlap weight, which is the probability of membership in the non-observed exposure group (one minus the propensity of in the observed group $)^{33,34}$. We then-for all outcome models-assessed covariate balance, calculating the standardized difference after application of the overlap weight for all predefined variables, 100 algorithmically selected high-dimensional variables, and all high-dimensional variables that were not selected for inclusion in the propensity score models. We present the distribution of these standardized differences for 20 randomly selected outcome-specific cohorts, and across all outcomes, and the covariate distributions in overall cohort after adjustment.

The risks of health resource use-including outpatient encounter and death between individuals with COVID-19 and all VHA users, and between COVID-19 hospitalization and influenza hospitalization-were estimated from a Cox survival model weighted by overlap weights, in which death was considered as a competing risk in the evaluation of health resource use. The frequency of outpatient encounter was modelled on the basis of a weighted linear regression. Hazard ratios for each of the outcomes-including incident diagnoses, incident medication use and incident laboratory abnormalities-were estimated from cause-specific hazard models weighted by overlap weights, in which occurrence of death was considered as a competing risk. Event rates per 1,000 participants at 6 months (180 days) of follow-up in each group, and the adjusted excess burden based on the differences between two groups, were estimated. Models were built only for outcomes that occurred in at least 10 participants from each group. A Bonferroni correction was applied in consideration of multiple hypotheses testing for high-dimensional outcomes. A $P$ values of less than $6.57 \times 10^{-5}$ was considered statistically significant. Results are additionally presented with a focus on identified post-acute sequelae of COVID-19, in which we selected those sequelae with a hazard ratio greater than 1 and $P$ values of less than $6.57 \times 10^{-5}$. High-dimensional analyses of individuals who were hospitalized for COVID-19 versus seasonal influenza, which were adjusted for the severity of the acute infection (through inclusion of SOFA scores), were additionally undertaken. In addition, high-dimensional analyses were also conducted to evaluate the risk of six-month clinical outcomes in people who were hospitalized for COVID-19 versus those who were hospitalized for other causes. Participants who were hospitalized for other causes who survived the first 30 days after hospital admission were enrolled between 1 October 2016 and 29 February $2020(n=901,516)$.

We examined the risk of high-resolution, prespecified outcomes across care settings of the acute phase of the disease, analysing differences in risk of clinical manifestations of post-acute COVID-19 between mutually exclusive groups of people who were positive for COVID-19 (non-hospitalized, hospitalized and admitted to intensive care), and VHA users who were not positive for COVID-19. Propensity scores for group membership were estimated in outcome-specific cohorts free of the related disease at baseline ${ }^{32}$. Standardized differences in the predefined and algorithmically selected high-dimensional covariates are presented after application of overlap weighting ${ }^{35}$. The percentage of outcomes ascertained in the COVID-19 group in an inpatient and outpatient setting are presented. We then constructed Cox survival models to analyse the risk of outcomes using overlap weighting for multiple treatments. We report hazard ratios and event rate differences between each group. We also estimated the risks of prespecified outcomes among individuals who were hospitalized with COVID-19 or seasonal influenza, which were additionally adjusted using SOFA scores.

All analyses were done using SAS Enterprise Guide version 7.1. Data visualizations were performed in $\mathrm{R}$ 4.0.3. The study was approved by the Institutional Review Board of the Department of Veterans Affairs St. Louis Health Care System.

\section{Reporting summary}

Further information on research design is available in the Nature Research Reporting Summary linked to this paper.

\section{Data availability}

The data that support the findings of this study are available from the VA. VA data are made freely available to researchers behind the VA firewall with an approved VA study protocol. More information is available at https://www.virec.research.va.gov or by contacting the VA Information Resource Center (VIReC) at VIReC@va.gov.

\section{Code availability}

SAS and R programming codes are available at https://github.com/ yxie618/HDlongCOVID.

10. Xie, Y. et al. Proton pump inhibitors and risk of incident CKD and progression to ESRD. J. Am. Soc. Nephrol. 27, 3153-3163 (2016).

11. Xie, Y. et al. Risk of death among users of proton pump inhibitors: a longitudinal observational cohort study of United States veterans. BMJ Open 7, e015735 (2017).

12. Xie, Y. et al. Long-term kidney outcomes among users of proton pump inhibitors without intervening acute kidney injury. Kidney Int. 91, 1482-1494 (2017).

13. Xie, Y. et al. Higher blood urea nitrogen is associated with increased risk of incident diabetes mellitus. Kidney Int. 93, 741-752 (2018).

14. Vincent, B. M., Wiitala, W. L., Burns, J. A., Iwashyna, T. J. \& Prescott, H. C. Using Veterans Affairs corporate data warehouse to identify 30-day hospital readmissions. Health Serv. Outcomes Res. Methodol. 18, 143-154 (2018).

15. Kind, A. J. H. \& Buckingham, W. R. Making neighborhood-disadvantage metrics accessible - the neighborhood atlas. N. Engl. J. Med. 378, 2456-2458 (2018).

16. Lipsitch, M., Tchetgen Tchetgen, E. \& Cohen, T. Negative controls: a tool for detecting confounding and bias in observational studies. Epidemiology 21, 383-388 (2010).

17. Shi, X., Miao, W. \& Tchetgen, E. T. A selective review of negative control methods in epidemiology. Curr. Epidemiol. Rep. 7, 190-202 (2020).

18. Wei, Y. et al. Short term exposure to fine particulate matter and hospital admission risks and costs in the Medicare population: time stratified, case crossover study. Br. Med. J. 367, 16258 (2019).

19. Aubert, C. E. et al. Best definitions of multimorbidity to identify patients with high health care resource utilization. Mayo Clin. Proc. Innov. Qual. Outcomes 4, 40-49 (2020).

20. HCUP CCSR. Healthcare Cost and Utilization Project (HCUP) (Agency for Healthcare Research and Quality, 2020).

21. Olvey, E. L., Clauschee, S. \& Malone, D. C. Comparison of critical drug-drug interaction listings: the Department of Veterans Affairs medical system and standard reference compendia. Clin. Pharmacol. Ther. 87, 48-51 (2010).

22. Greene, M. Steinman, M. A. McNicholl, I. R. \& Valcour, V. Polypharmacy, drug-drug interactions, and potentially inappropriate medications in older adults with human immunodeficiency virus infection. J. Am. Geriatr. Soc. 62, 447-453 (2014).

23. Xie, Y. et al. Estimates of all cause mortality and cause specific mortality associated with proton pump inhibitors among US veterans: cohort study. Br. Med. J. 365, l1580 (2019).

24. Xie, Y. et al. Comparative effectiveness of SGLT2 inhibitors, GLP-1 receptor agonists, DPP-4 inhibitors, and sulfonylureas on risk of kidney outcomes: emulation of a target trial using health care databases. Diabetes Care 43, 2859-2869 (2020).

25. Xie, Y. et al. Comparative effectiveness of the sodium-glucose cotransporter 2 inhibitor empagliflozin versus other antihyperglycemics on risk of major adverse kidney events. Diabetes Care 43, 2785-2795 (2020).

26. Bowe, B. et al. Acute kidney injury in a national cohort of hospitalized US veterans with COVID-19. Clin. J. Am. Soc. Nephrol. 16, 14-25 (2021).

27. Bowe, B. et al. The 2016 global and national burden of diabetes mellitus attributable to $\mathrm{PM}_{2.5}$ air pollution. Lancet Planet. Health 2, e301-e312 (2018). 


\section{Article}

28. Bowe, B., Xie, Y., Xian, H., Balasubramanian, S. \& Al-Aly, Z. Low levels of high-density lipoprotein cholesterol increase the risk of incident kidney disease and its progression. Kidney Int. 89, 886-896 (2016)

29. Bowe, B. et al. High density lipoprotein cholesterol and the risk of all-cause mortality among U.S. veterans. Clin. J. Am. Soc. Nephrol. 11, 1784-1793 (2016)

30. Vincent, J. L. et al. The SOFA (sepsis-related organ failure assessment) score to describe organ dysfunction/failure. on behalf of the Working Group on Sepsis-Related Problems of the European Society of Intensive Care Medicine. Intensive Care Med. 22, 707-710 (1996).

31. Vincent, J. L. et al. Use of the SOFA score to assess the incidence of organ dysfunction/ failure in intensive care units: results of a multicenter, prospective study. Crit. Care Med. 26, 1793-1800 (1998)

32. McCaffrey, D. F. et al. A tutorial on propensity score estimation for multiple treatments using generalized boosted models. Stat. Med. 32, 3388-3414 (2013).

33. Thomas, L. E., Li, F. \& Pencina, M. J. Overlap weighting: a propensity score method that mimics attributes of a randomized clinical trial. J. Am. Med. Assoc. 323, 2417-2418 (2020).

34. Li, F., Morgan, K. L. \& Zaslavsky, A. M. Balancing covariates via propensity score weighting. J. Am. Stat. Assoc. 113, 390-400 (2018).

35. Li, F. \& Li, F. Propensity score weighting for causal inference with multiple treatments. Ann. Appl. Stat. 13, 2389-2415 (2019)

Acknowledgements This study used data from the VA COVID-19 Shared Data Resource. M. Cai developed the data visualization and A. K. Gibson provided technical and editorial assistance. This research was funded by the United States Department of Veterans Affairs and the Institute for Public Health at Washington University in Saint Louis (for Z.A.-A.), and two American Society of Nephrology and KidneyCure fellowship awards (for $Y . X$. and B.B.). The funders of this study had no role in study design; collection, analysis and interpretation of data; writing the report; and the decision to submit the report for publication. This research project was reviewed and approved by the Institutional Review Board of the Department of Veterans Affairs Saint Louis Health Care System.

Author contributions Z.A.-A., Y.X. and B.B. contributed to the development of the stud concept and design. Y.X. and B.B. contributed to data acquisition. Z.A.-A., Y.X. and B.B. contributed to data analysis and interpretation. Y.X. and B.B. contributed to statistical analysis. Z.A.-A. and Y.X. drafted the manuscript. Z.A.-A., Y.X. and B.B. contributed to critical revision of the manuscript. Z.A.-A. provided administrative, technical and material support. Z.A.-A. provided supervision and mentorship. Each author contributed important intellectual content during manuscript drafting or revision, and accepts accountability for the overall work by ensuring that questions pertaining to the accuracy or integrity of any portion of the work are appropriately investigated and resolved. All authors approved the final version of the report. The corresponding author attests that all the listed authors meet the authorship criteria and that no others meeting the criteria have been omitted.

Competing interests The authors declare no competing interests.

Additional information

Supplementary information The online version contains supplementary material available at https://doi.org/10.1038/s41586-021-03553-9.

Correspondence and requests for materials should be addressed to Z.A.-A.

Peer review information Nature thanks Paul Garner, Sachin Yende and the other, anonymous, reviewer(s) for their contribution to the peer review of this work.

Reprints and permissions information is available at http://www.nature.com/reprints. 

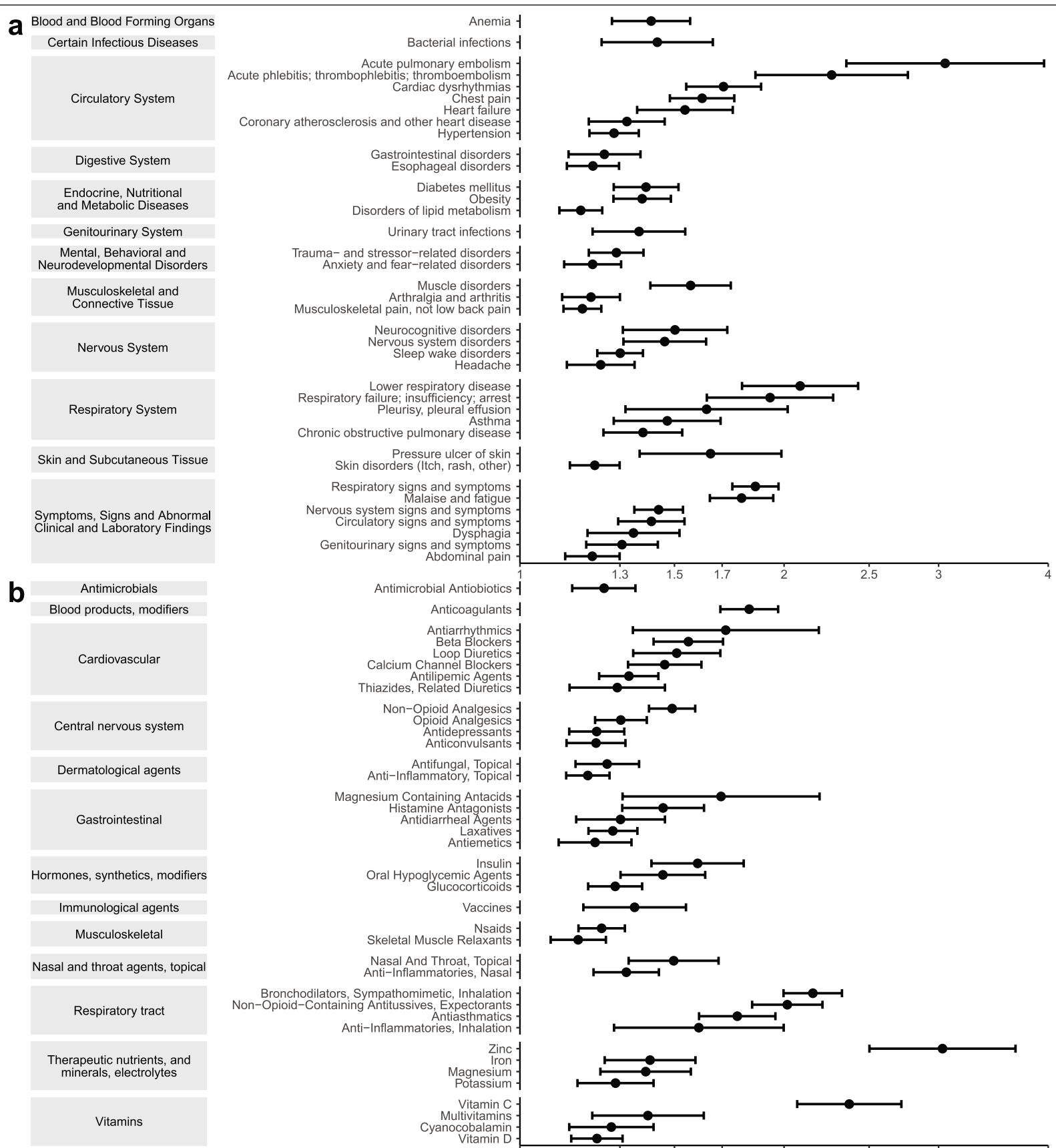
a

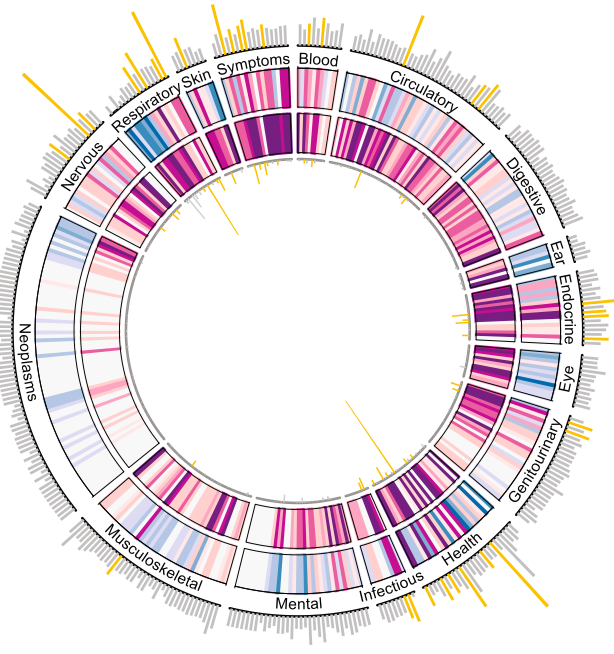

b

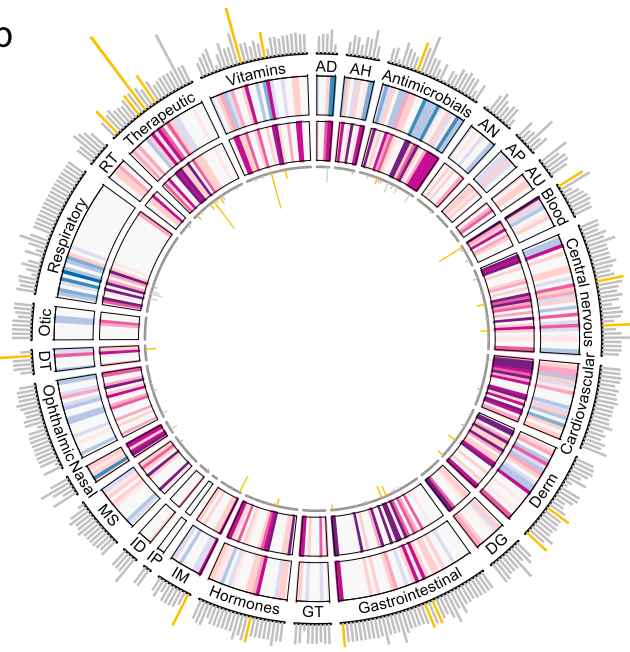

C

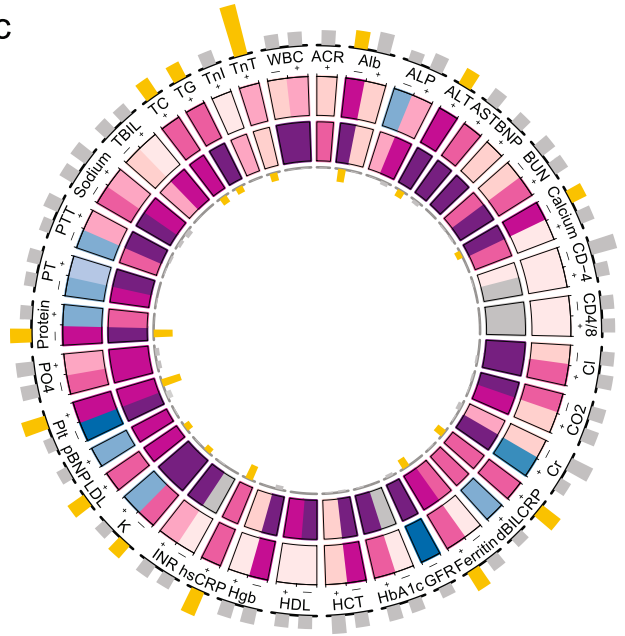

d

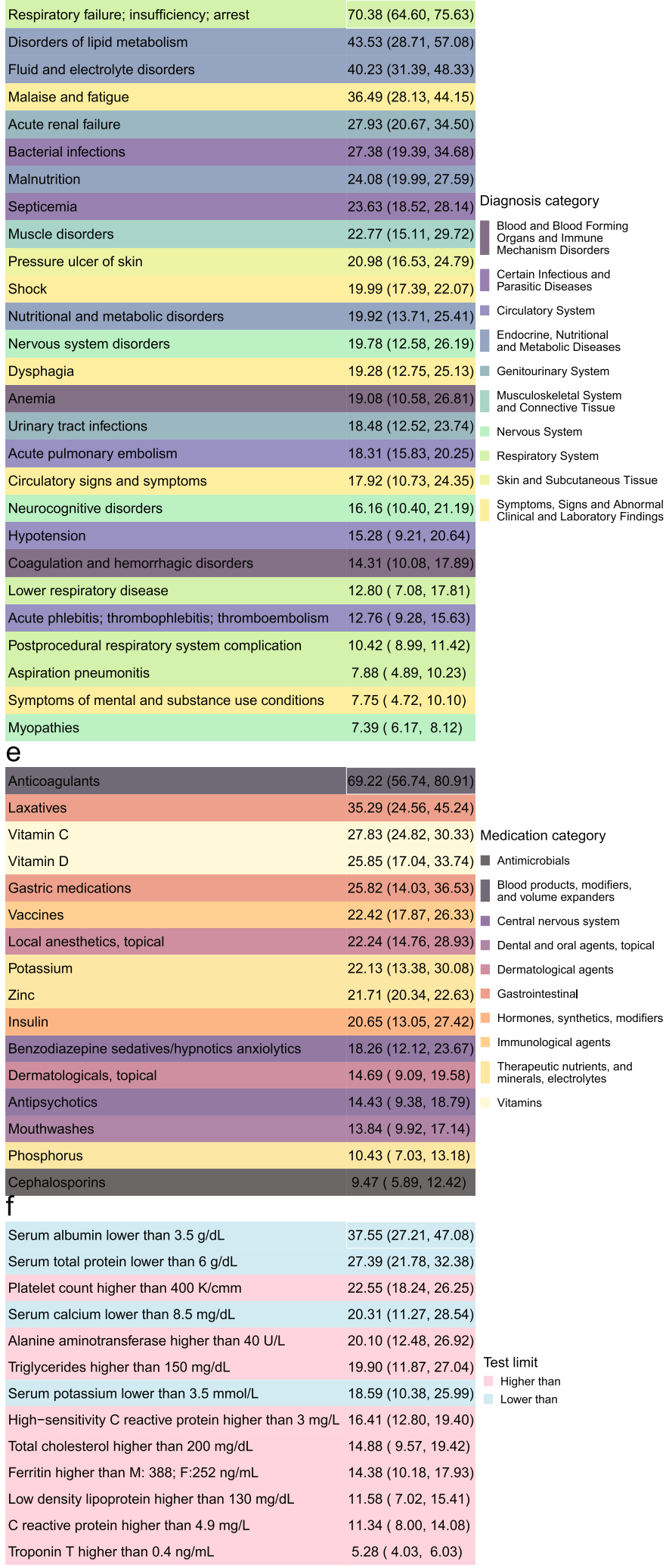

Extended Data Fig. 2 |See next page for caption. 
Extended Data Fig. 2 | High-dimensional identification of the incident postacute sequelae in people who were hospitalized for COVID-19. $a-f$, incident diagnoses (a, d), incident medication use (b,e) and incident laboratory abnormalities (c,f). Individuals who were hospitalized with seasonal influenza served as the referent category. Post-acute sequelae were ascertained from 30 days after infection until the end of follow-up. a-c, Beginning from the outside ring, the first ring represents hazard ratios for the post-acute sequelae of COVID-19. A higher bar indicates a larger hazard ratio. Hazard ratios with a point estimate larger than one and that are statistically significant are coloured in yellow. The second ring represents excess burden per 1,000 patients with COVID-19 at 6 months. Colour of the cell indicates value of the excess burden; deeper shades of red indicate higher excess burden and deeper shades of blue indicate greater reduced burden. The third ring represents the baseline incident rate in the control group; deeper shades of red indicate higher incident rate. The fourth ring represents negative log of the $P$ value; a higher bar indicates a smaller $P$ value and yellow indicates statistically significant. d-f, Sequelae were selected based on having a hazard ratio larger than one and $P$ value less than $6.57 \times 10^{-5}$. Excess burdens per 1,000 patients with COVID-19 at 6 months are presented. Within each domain, outcomes are ranked based on excess burden from high to low. Diagnoses are coloured based on diagnosis group, medications are coloured based on medication class, and laboratory abnormalities are coloured based on higher or lower than normal range. 
a Blood and Blood Forming Organs
and Immune Mechanism Disorders

Certain Infectious and

Parasitic Diseases

Circulatory System

Endocrine, Nutritional

and Metabolic Diseases

Genitourinary System

Musculoskeletal and Connective Tiss

Nervous System

Skin and Subcutaneous Tissue

Symptoms, Signs and Clinical and Laboratory Findings

b

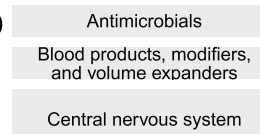

Dental and oral agents, topical

Dermatological agents

Gastrointestinal

Hormones, synthetics, modifiers

Immunological agents

Therapeutic nutrients, and minerals, electrolytes

Vitamins

\section{C}

Laboratory result
higher than threshold

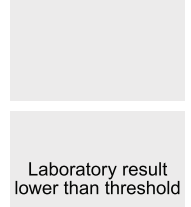

Coagulation and hemorrhagic disorders

Anemia

Septicemia

Bacterial infections

Acute pulmonary embolism

Acute phlebitis; thrombophlebitis; thromboembolism

Hypotension

Malnutrition

Fluid and electrolyte disorders

Nutritional and metabolic disorders

Disorders of lipid metabolism

Acute renal failure

Urinary tract infections -

Muscle disorders

Myopathies

Neurocognitive disorders

Nervous system disorders

Postoperative respiratory system complication

Respiratory failure; insufficiency; arrest

Aspiration pneumonitis

Lower respiratory disease

Pressure ulcer of skin -

Shock

Symptoms of mental and substance use conditions

Malaise and fatigue

Dysphagia

Circulatory signs and symptoms

Cephalosporins -

Anticoagulants -

Antipsychotics

Benzodiazepine Sedatives, Hypnotics Anxiolytics

Mouthwashes

Dermatologicals, Topical

Local Anesthetics, Topical -

Laxatives -

Gastric Medications -

Insulin $f$

Vaccines $f$

$$
\text { Zinc }
$$

Phosphorus

Potassium

Vitamin C

Vitamin D

Troponin T

High-sensitivity $\mathrm{C}$ reactive protein

Platelet count

$C$ reactive protein

Ferritin

Low density lipoprotein cholesterol

Total cholesterol

Triglycerides

Alanine aminotransferase

Serum total protein

Serum albumin

Serum potassium

Serum calcium
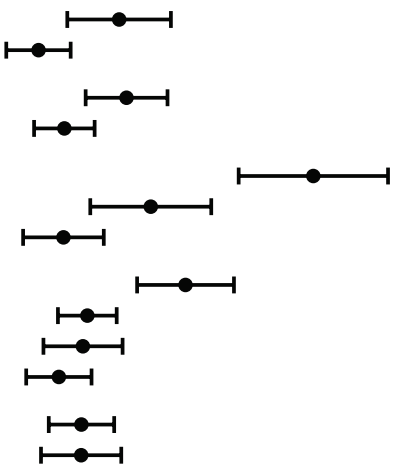

$\longmapsto$
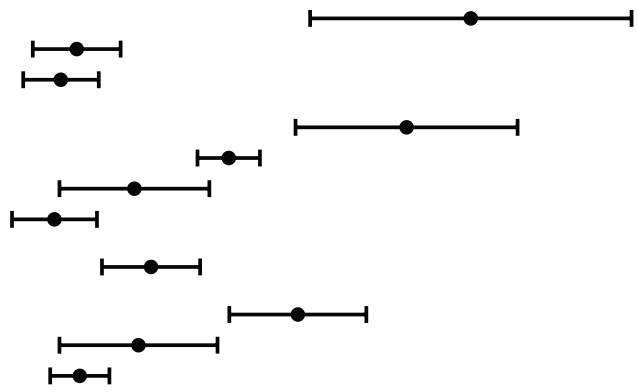

$\longrightarrow$

$\longmapsto$

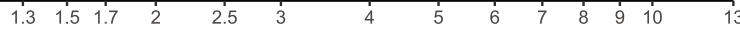

$\stackrel{\longrightarrow}{\longrightarrow}$
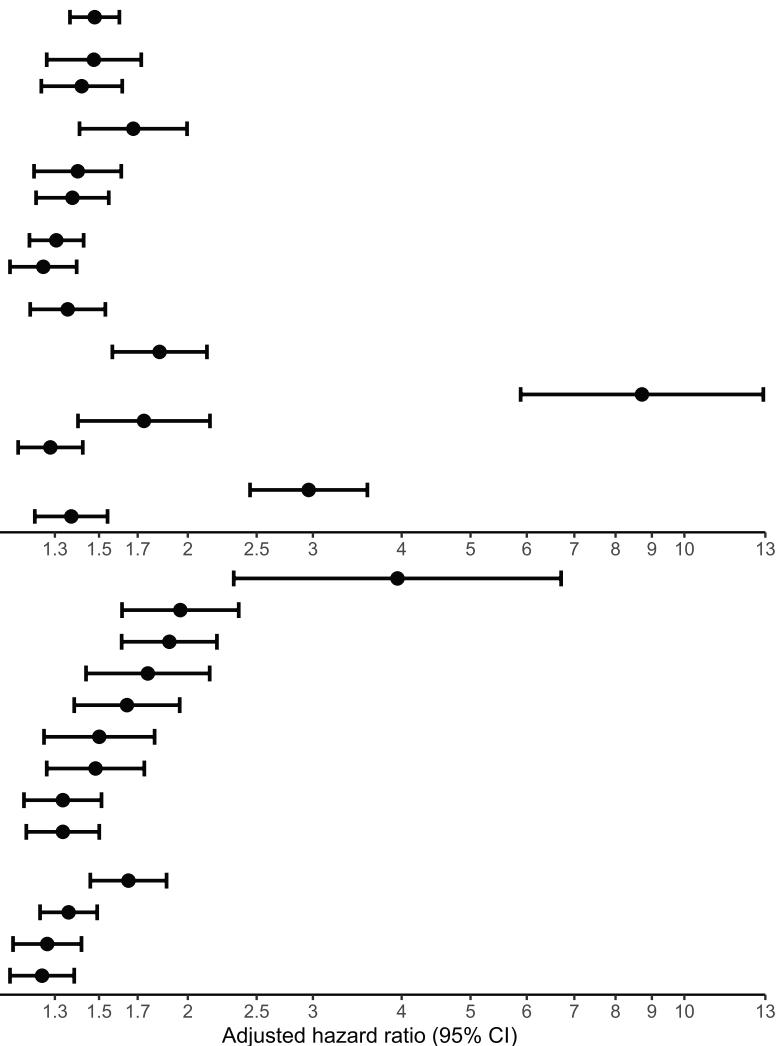

Outcomes were ascertained from day 30 after hospital admission until the end of follow-up. Adjusted hazard ratios for incident sequelae that are larger than 1 and $P$ value less than $6.57 \times 10^{-5}$ are presented. Hazard ratios (dots) and $95 \%$ confidence intervals (bars) are presented on a $\log _{10}$ scale.
Extended Data Fig. 3 | Risk of incident post-acute sequelae in people who were hospitalized for COVID-19. a-c, Incident diagnoses (a), incident medication use (b) and incident laboratory abnormalities (c). People who were hospitalized with seasonal influenza served as the referent category. 

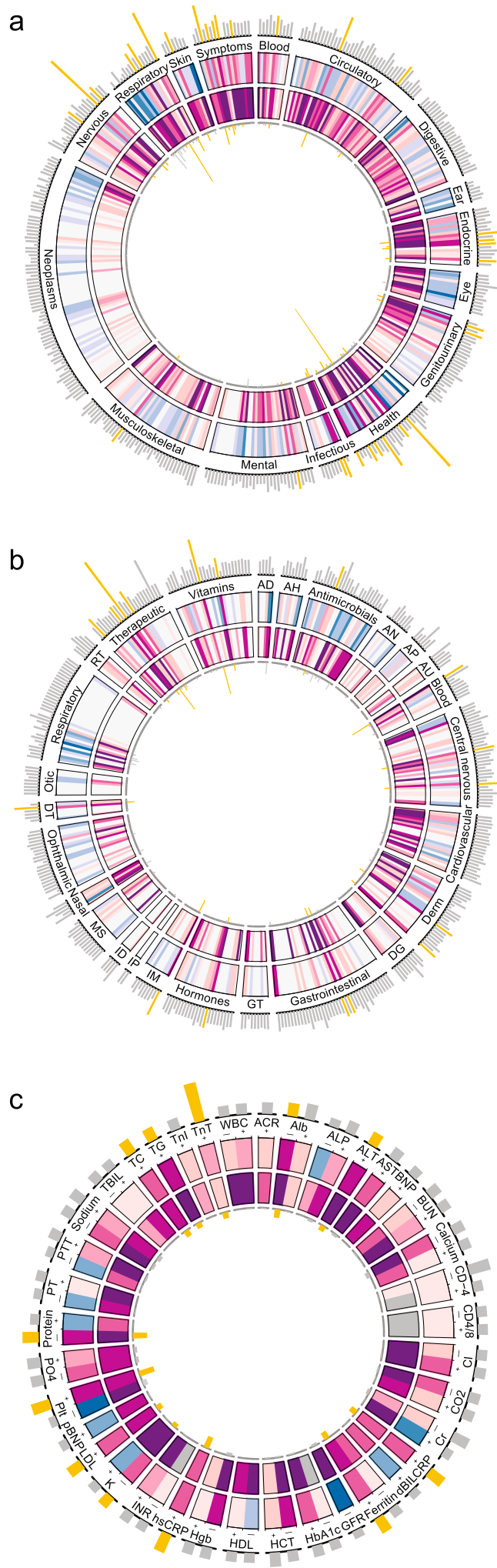

d

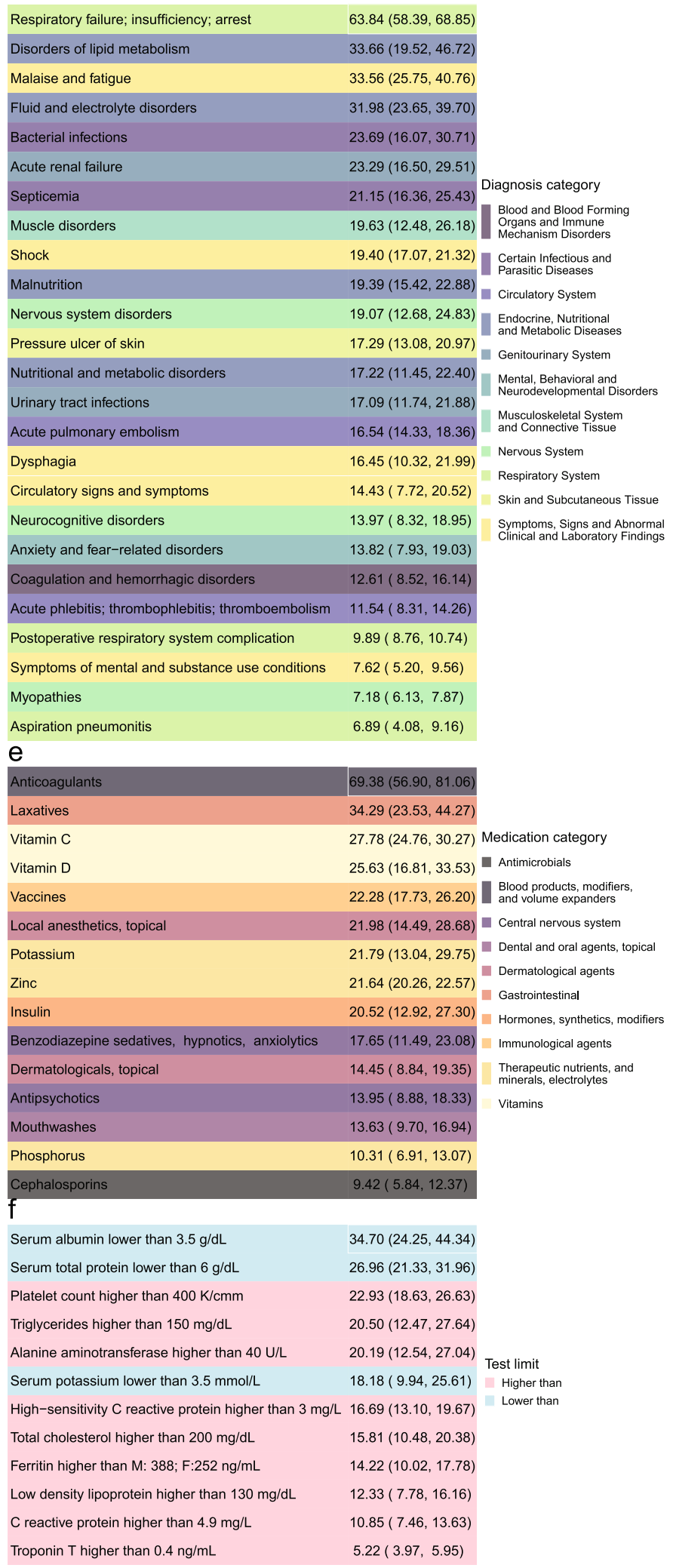

Extended Data Fig. 4 |See next page for caption. 


\section{Article}

Extended Data Fig. 4 | High-dimensional identification of the incident post-acute sequelae in people who were hospitalized for COVID-19 after additionally adjusting for severity of the acute infection. a-f, Incident diagnoses (a, d), incident medication use (b,e) and incident laboratory abnormalities $(\mathbf{c}, \mathbf{f})$. Individuals who were hospitalized with seasonal influenza served as the referent category. Post-acute sequelae were ascertained from 30 days after infection until the end of follow-up. a-c, Beginning from the outside ring, the first ring represents hazard ratios for the post-acute sequelae of COVID-19. A higher bar indicates a larger hazard ratio. Hazard ratios with a point estimate larger than 1 and that are statistically significant are coloured in yellow. The second ring represents excess burden per 1,000 patients with COVID-19 at 6 months. The colour of the cell indicates the value of the excess burden; deeper shades of red indicate higher excess burden and deeper shades of blue indicate greater reduced burden. The third ring represents the baseline incident rate in the control group; deeper shades of red indicate higher incident rate. The fourth ring represents negative log of the $P$ value; a higher bar indicates smaller $P$ value and yellow bar indicates statistically significant. d-f, Sequelae were selected based on hazard ratio larger than 1 and $P$ value less than $6.57 \times 10^{-5}$. Excess burdens per 1,000 patients with COVID-19 at 6 months are presented. Within each domain, outcomes are ranked based on excess burden from high to low. Diagnoses are coloured based on diagnosis group, medications are coloured based on medication class, and laboratory abnormalities are coloured based on higher or lower than normal range. 


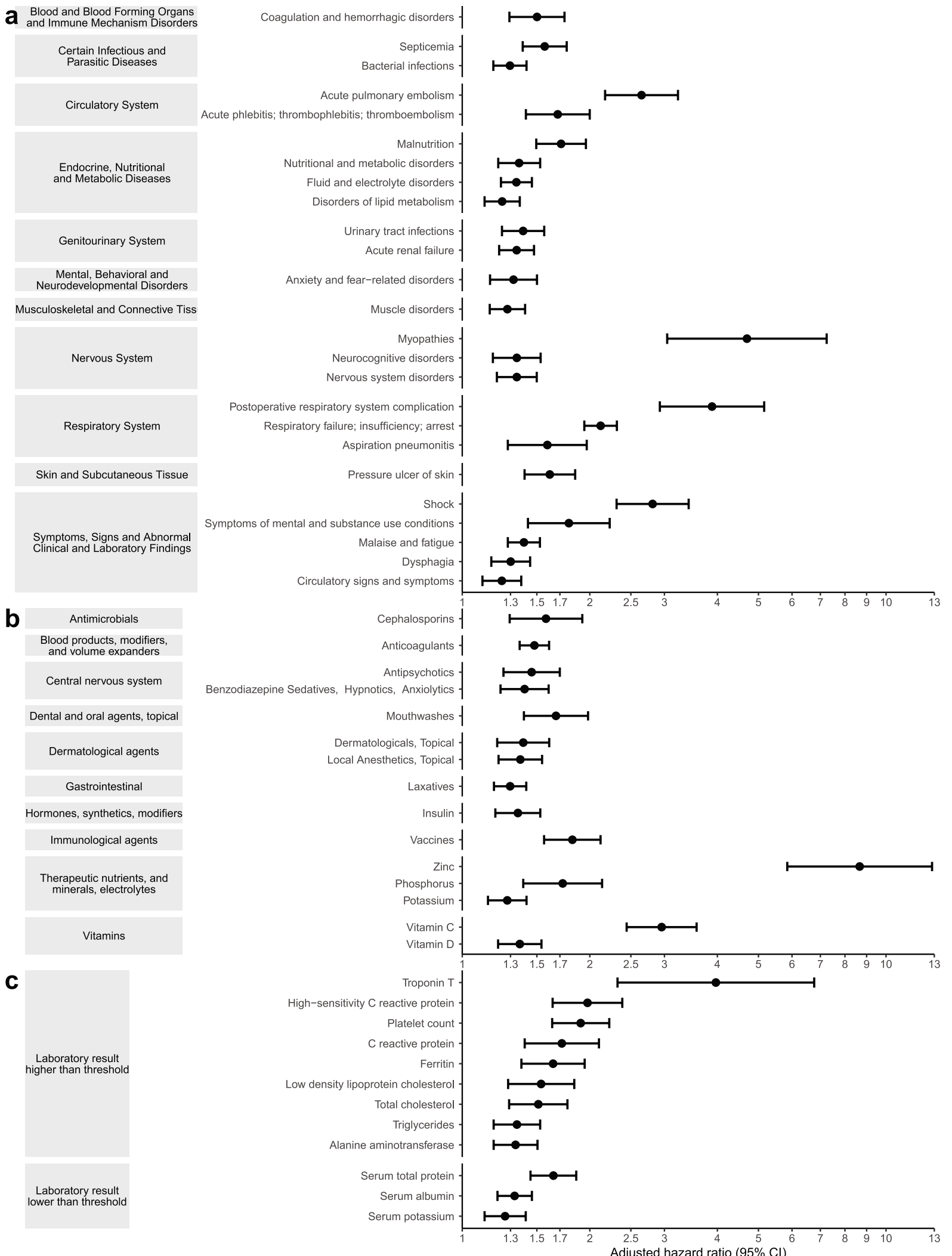

Extended Data Fig. $5 \mid$ Risk of incident post-acute sequelae in people who were hospitalized for COVID-19 after additionally adjusting for severity of the acute infection. a-c, Incident diagnoses (a), incident medication use (b) and incident laboratory abnormalities (c). People who had been hospitalized with seasonal influenza served as the referent category. Outcomes were

ascertained from day 30 after hospital admission until the end of follow-up. Adjusted hazard ratios for incident sequelae that are larger than 1 and $P$ value less than $6.57 \times 10^{-5}$ are presented. Hazard ratios (dots) and $95 \%$ confidence intervals (bars) are presented on $\log _{10}$ scale. 
a
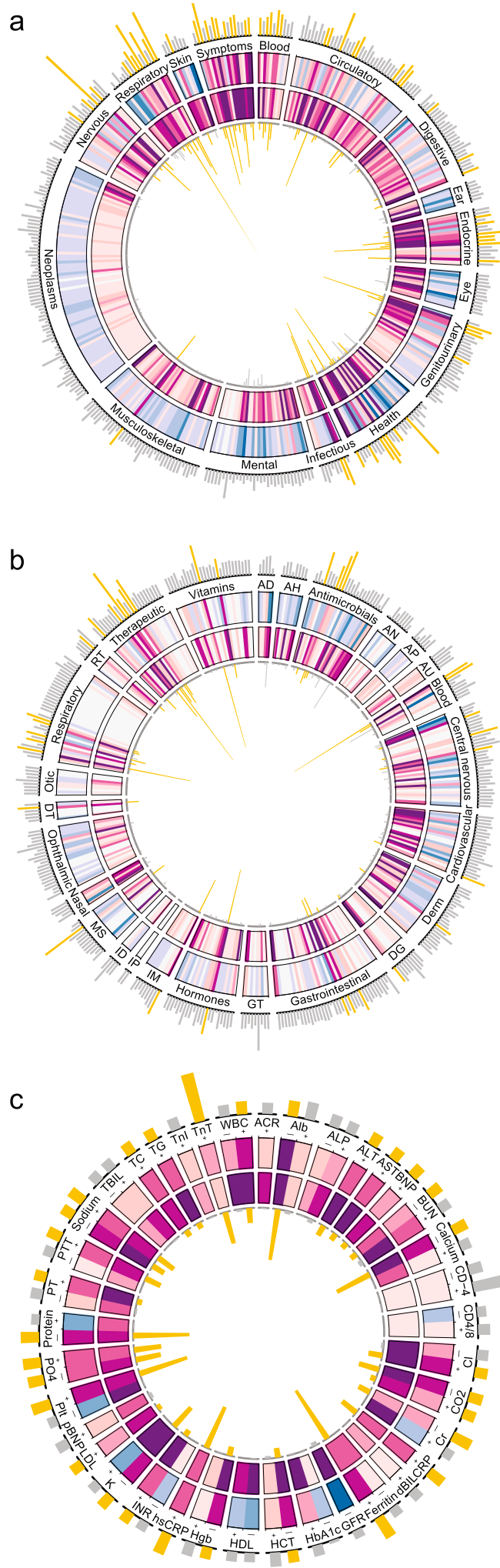

d

Respiratory failure; insufficiency; arrest

$\quad 85.43(83.47,87.28)$ Rlupictory signs and symptoms $\quad 58.99(53.09,64.59)$

Malaise and fatigue disorders $\quad 50.99(46.70,55.05)$

Acute renal failure $\quad 43.66(40.41,46.72)$

\begin{tabular}{ll} 
Bacterial infections & $40.76(36.87,44.43)$ \\
Disorders of lipid metabolism & $39.18(30.04,47.82)$ \\
\hline
\end{tabular}

$\begin{array}{ll}\text { Septicemia } & 31.37(29.08,33.49) \\ \text { Muscle disorders } & 31.31(27.48,34.91)\end{array}$

$\begin{array}{ll}\text { Anemia } & 25.37(20.70,29.77) \\ \text { Nervous system signs and symptoms } & 24.79(18.19,31.06) \\ & 23.88(20.43,27.09)\end{array}$

Nutritional and metabolic disorders $\quad 23.20(19.97,26.20)$ Blood and Blood Forming

Circulatory signs and symptoms $\quad 22.89(19.03,26.48)$

Lower respiratory disease $\quad 22.89(20.44,25.13)$

22.89 (20.44, 25.13) Certain Infectious and

Hypertension $22.56(17.69,27.13)$ Certain Infectious and

$\begin{array}{ll}\text { Nervous system disorders } & 20.50(16.21,24.51) \\ \text { Malnutrition } & 19.91(17.55,22.07)\end{array}$

\begin{tabular}{ll} 
Malnutrition & $19.91(17.55,22.07)$ \\
Urinary tract infections & $19.70(16.30,22.85$ \\
\hline
\end{tabular}

$\begin{array}{ll}\text { Shock } & 19.70(16.30,22.85) \\ \text { Shact infections } & 19.31(18.13,20.36)\end{array}$

Hypotension $18.66(15.53,21.56)$

$\begin{array}{ll}\text { Acute pulmonary embolism } & 18.63(17.48,19.66) \\ \text { Cardiac dysrhythmias } & 17.22(12.53,21.61)\end{array}$

$\begin{array}{ll}\text { Cardiac dysrhythmias } & 17.22(12.53,21.61) \\ \text { Coagulation and hemorrhagic disorders } & 16.98(15.03,18.75)\end{array}$

$\begin{array}{ll}\text { Coagulation and hemorrhagic disorders } & 16.81(10.01,23.22)\end{array}$

Diabetes mellitus $\quad 15.71(11.04,19.94)$

$\begin{array}{ll}\text { Gastrointestinal disorders } & 15.48(11.12,19.54) \\ \text { Neurocognitive disorders } & 14.48(11.00,17.68)\end{array}$

Pressure ulcer of skin $\quad 14.45(11.62,17.05)$

\begin{tabular}{ll} 
Diseases of white blood cells & $14.45(12.08,16.60)$ \\
\hline
\end{tabular}

\begin{tabular}{ll} 
Diseases of white blood celis & $14.45(12.08,16.60)$ \\
\hline Esophageal disorders & $13.47(7.64,18.94)$ \\
\hline
\end{tabular}

Chronic obstructive pulmonary disease $\quad 12.35(7.79,16.58)$

Chronic kidney disease $\quad 11.98(7.94,15.70)$

Obesity $\quad 11.98(7.94,15.69)$

Postprocedural respiratory system complication $\quad 10.07(9.43,10.61)$

Fever $\quad 9.30(7.50,10.88)$

Aspiration pneumonitis $\quad 7.85(6.32,9.18)$

$\begin{array}{ll}\text { Gastrointestinal hemorrhage } & 7.27(4.66,9.62) \\ \text { Myopathies } & 6.99(6.59,7.31)\end{array}$

Myopathies

$6.84(4.66,8.76)$

$\begin{array}{ll}\text { Pleurisy, pleural effusion } & 6.46(3.66,8.98) \\ \text { Symptoms of mental and substance use conditions } & 6.16(4.40,7.69) \\ \text { Pneumothorax } & 3.89(3.29,4.37)\end{array}$

\begin{tabular}{ll} 
Pneumothorax & $3.89(3.29,4.37)$ \\
\hline
\end{tabular}

e

Anticoagulants $93.50(87.62,99.15)$ Medication category

Bronchodilators, sympathomimetic, inhalation $\quad 47.49(43.14,51.57)$

Insulin $\quad 36.51(32.96,39.82)$

Laxatives $\quad 32.54(26.15,38.63)$

$\begin{array}{ll}\text { Potassium } & 31.54(27.21,35.61) \\ \text { Vitamin C } & 24.97(23.37,26.44)\end{array}$

$\begin{array}{ll}\text { Vitamin D } & 23.67(18.18,28.82) \\ \text { Non-opioid analgesics } & 22.67(11.90,32.99) \\ \text { Vaccings } & 22.36(19.65,24.85)\end{array}$

Vaccines $\quad 22.36(19.65,24.85)$

$\begin{array}{ll}\text { Zinc } & 21.40(20.95,21.80) \\ \text { Histamine antagonists } & 18.66(14.64,22.39)\end{array}$

$\begin{array}{ll}\text { Histamine antagonists } & 17.96(14.54,21.13) \\ \text { Magnesium } & 14.66(9.10,18.69)\end{array}$

Non-opioid-containing antitussives/expectorants $\quad 14.06(9.10,18.69)$

Local anesthetics, topical $\quad 13.79(9.08,18.18)$

Benzodiazepine sedatives/hypnotics anxiolytics $\quad 12.79(8.96,16.34)$

Phosphorus $\quad 10.62(8.98,12.07)$

$\begin{array}{lc}\text { Mouthwashes } & 10.33(7.88,12.54) \\ \text { Cephalosporins } & 9.62(7.58,11.43) \\ \text { Thrombolytics } & 5.04(4.06,5.85)\end{array}$

\begin{tabular}{ll} 
Thrombolytics & $5.04(4.06,5.85)$ \\
Cardiovascular agents & $4.73(3.33,5.92)$ \\
\hline
\end{tabular}

Bicarbonates $3.91(3.20,4.46)$

$\begin{array}{ll}\text { Mucolytics } & 2.59(1.64,3.34) \\ \text { Extended spectrum penicillins } & 2.10(1.49,2.54)\end{array}$

f

Serum albumin lower than $3.5 \mathrm{~g} / \mathrm{dL}$
Hematocrit lower than M: $42 ; \mathrm{F}: 37 \%$

Hemoglobin lower than M: $14 ; \mathrm{F}: 12 \mathrm{~g} / \mathrm{d}$

Serum calcium lower than $8.5 \mathrm{mg} / \mathrm{d}$

Serum total protein lower than $6 \mathrm{~g} / \mathrm{dL}$

Serum potassium lower than $3.5 \mathrm{mmo} /$

Serum sodium lower than $136 \mathrm{mmo} / \mathrm{L}$

Carbon dioxide lower than $22 \mathrm{mmol} / \mathrm{L}$

Total white blood cell count higher than $10.8 \mathrm{~K} / \mathrm{cmm}$

Platelet count higher than $400 \mathrm{~K} / \mathrm{cmm}$

Ferritin higher than M: $388 . \mathrm{F} \cdot 252 \mathrm{ng} / \mathrm{mL}$

Serum phosphate lower than $25 \mathrm{mg} / \mathrm{dL}$

Alanine aminotransferase higher than $40 \mathrm{U} / \mathrm{L}$

culatory System

Digestive System

Endocrine, Nutritional
and Metabolic Diseases

Genitourinary System

Musculoskeletal System
and Connective Tissue

Nervous System

Respiratory System

Skin and Subcutaneous Tissue

Symptoms, Signs and Abnormal
Clinical and Laboratory Findings

Antimicrobials

Blood products, modifiers,

Cardiovascular

Central nervous system

Dental and oral agents, topical

Dermatological agents

Gastrointestinal

Hormones, synthetics, modifiers

Respiratory tract

Therapeutic nutrients, and
minerals, electrolytes

Vitamins

Partial thromboplastin time higher than 36.5 SEC

Serum phosphate higher than $4.9 \mathrm{mg} / \mathrm{dL}$

Triglycerides higher than $150 \mathrm{mg} / \mathrm{dL}$

Serum sodium higher than $145 \mathrm{mmol} / \mathrm{L}$

Aspartate aminotransferase higher than $40 \mathrm{U} / \mathrm{L}$

. 1.2 ratio

C reactive protein higher than $4.9 \mathrm{mg} / \mathrm{L}$

Total cholesterol higher than $200 \mathrm{mg} / \mathrm{dL}$

Protriur

Catiurelic peplide higher than $100 \mathrm{pg} / \mathrm{mL}$

ow density lipoprotein higher than $130 \mathrm{mg}$

Blood urea nitrogen lower than $7 \mathrm{mg} / \mathrm{dL}$

Troponin T higher than $0.4 \mathrm{ng} / \mathrm{mL}$

Serum creatinine lower than $0.5 \mathrm{mg} / \mathrm{dL}$

(9.25 $(43.92,54.31)$ $37.76(26.66,48.37)$ $35.11(24.50,45.22)$ $34.40(29.98,38.55)$ $29.79(27.00,32.39)$ $26.97(22.65,31.02$ $25.38(19.36,31.06)$ (25.02 $(20.51,29.24)$ ( (2). $20.26(18.58,21.78)$ $17.55(14.89,19.99)$ Test limit

$17.30(15.62,18.80) \quad$ Higher than

$15.93(12.73,18.87)$ Lower than

$14.43(12.43,16.23)$

$13.66(8.54,18.43)$

$12.83(10.57,14.88)$

$12.73(8.52,16.64)$

$11.34(7.22,15.15)$

$10.68(8.77,12.37)$

$10.07(5.89,13.94)$

$8.51(5.42,11.32)$

$7.43(4.52,10.06)$

$7.04(3.79,9.98)$

$6.71(4.44,8.72)$ $5.11(4.72,5.41)$ $5.04(4.00,5.90)$

Extended Data Fig. 6 | See next page for caption. 
Extended Data Fig. 6 High-dimensional identification of the incident post-acute sequelae of people who were hospitalized for COVID-19.

a-f, Incident diagnoses (a, d), incident medication use (b,e) and incident laboratory abnormalities $(\mathbf{c}, \mathbf{f})$. Individuals who were hospitalized for other causes served as the referent category. Post-acute sequelae were ascertained from 30 days after infection until the end of follow-up. a-c, Beginning from the outside ring, the first ring represents hazard ratios for the post-acute sequelae of COVID-19. A higher bar indicates a larger hazard ratio. Hazard ratios with a point estimate larger than one and that are statistically significant are coloured in yellow. The second ring represents excess burden per 1,000 patients with COVID-19 at 6 months. Colour of the cell indicates value of the excess burden; deeper shades of red indicate higher excess burden and deeper shades of blue indicate greater reduced burden. The third ring represents the baseline incident rate in the control group; deeper shades of red indicate higher incident rate. The fourth ring represents negative log of the $P$ value; a higher bar indicates smaller $P$ value and yellow bar indicates statistically significant. d-f, Sequelae were selected on the basis of a hazard ratio larger than one and $P$ value less than $6.57 \times 10^{-5}$. Excess burdens per 1,000 patients with COVID-19 at 6 months are presented. Within each domain, outcomes are ranked based on excess burden from high to low. Diagnoses are coloured based on diagnosis group, medications are coloured based on medication class, and laboratory abnormalities are coloured based on higher or lower than normal range. 


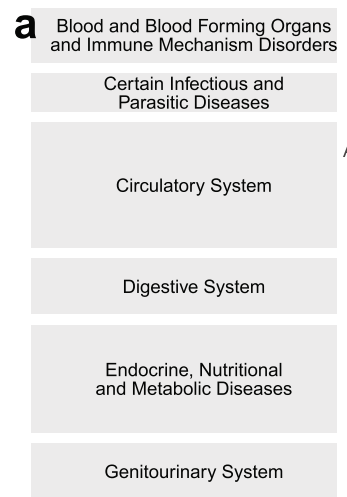

Musculoskeletal and Connective Tiss

Nervous System

Respiratory System

Skin and Subcutaneous Tissue

Symptoms, Signs and Abnormal Clinical and Laboratory Findings

b

\begin{tabular}{|c|}
\hline Antimicrobials \\
\hline $\begin{array}{c}\text { Blood products, modifiers, } \\
\text { and volume exbanders } \\
\text { Cardiovascular }\end{array}$ \\
\hline Central nervous system \\
\hline $\begin{array}{c}\text { Dental and oral agents, topical } \\
\text { Dermatological agents } \\
\text { Gastrointestinal }\end{array}$ \\
\hline $\begin{array}{c}\text { Hormones, synthetics, modifiers } \\
\text { Immunological agents }\end{array}$ \\
\hline Respiratory tract \\
\hline Therapeutic nutrients, and \\
minerals, electrolytes
\end{tabular}

C

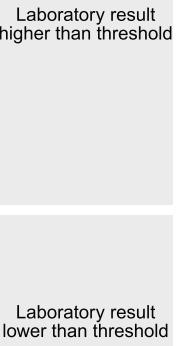

Coagulation and hemorrhagic disorders
Diseases of white blood cells

Anemia -1
Aneases of white blood cells

Septicemia
Bacterial infections

Acute pulmonary embolism

Acute phlebitis; thrombophlebitis; thromboembolism Cardiac arrest and ventricular fibrillation

Acute myocardial infarction Hypertension
Cardiac dysrhythmias

Gastrointestinal hemorrhage

Gastrointestinal disorders

Fluid and electrolyutrition Fluid and electrolyte disorders Disorders of lipid metabolism Acute renal failure Urinary tract infections
Chronic kidney disease

Muscle disorders 1

Myopathies

Neurocognitive disorders

Sivous system disorders

Postoperative respiratory system complication
Respiratory failure; insufficiency; arrest

Lower respiratory disease

Aspiration pneumonitis

Pleurisy, pleural effusion
Chronic obstructive pulmonary disease

Pressure ulcer of skin -

Shock

Malaise and fatigue
Respiratory signs and symptoms
Symptoms of mental and substance use conditions

and substance use conditions

Circulatory signs and symptoms

Other Beta-Lactams Antimicrobjals 1

Cephalosporins

Thrombolytics
Anticoagulants
-1

Cardiovascular Agents -

Benzodiazepine Sedatives Hypnotics Anxiolytics -
Non-Opioid Analgesics -

Mouthwashes -1

Local Anesthetics, Topical t

Laxatives -1

Insulin 1

Vaccines -

Bronchodilators, Sympathomimetic, Inhalation

Non-Opioid-Containing Antitussives, Expectorants -
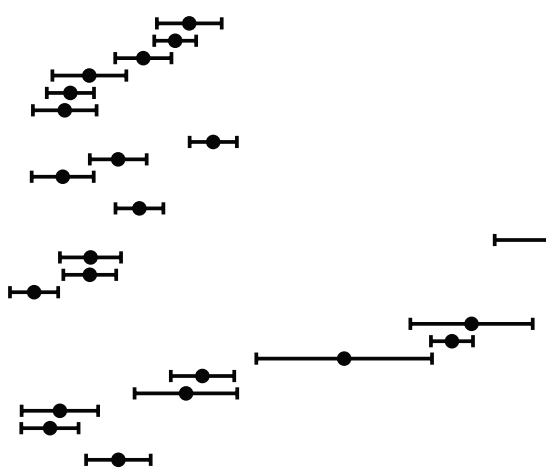

$\longmapsto$
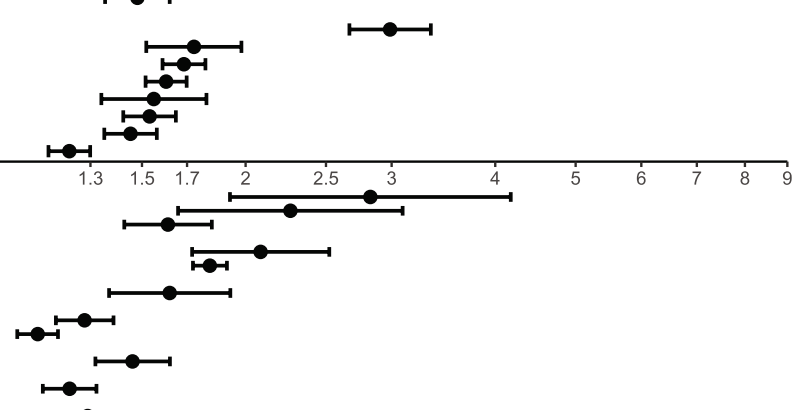

$\rightarrow$
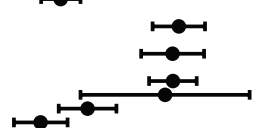

Bicarbonates

Potassium
Magnesium

Vitamin $C$

Troponin T High-sensitivity C reactive protein Platelet count

reactive protein Serum sodium Partial thromboplastin time Total white blood cell coun Serum chloride

$B$ natriuretic peptide

Carbon dioxide Total cholestero
Aspartate aminotransferase density lipoprotein cholestero Trigal normalized ratio Triglycerides
rothrombin time

Serum creatinine Serum creatinine
Serum total protein Serum phosphate Serum albumin Serum potassium Carbon dioxide Blood urea nitrogen Serum sodium Hematocrit Hemoglobin
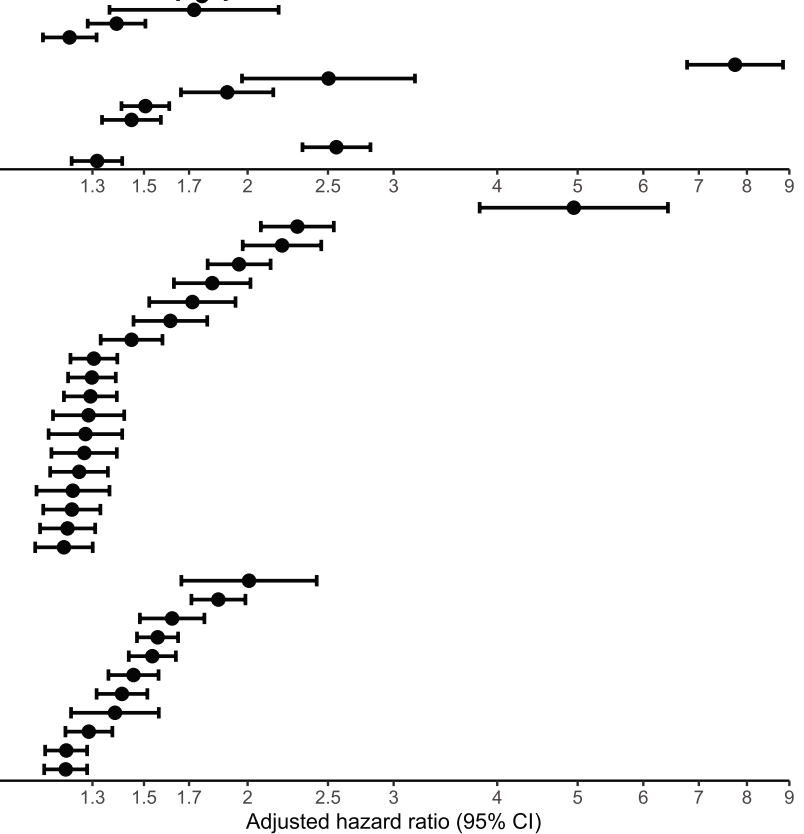

Outcomes were ascertained from day 30 after hospital admission until the end of follow-up. Adjusted hazard ratios for incident sequelae that are larger than 1 and $P$ value less than $6.57 \times 10^{-5}$ are presented. Hazard ratios (dots) and $95 \%$

confidence intervals (bars) are presented on $\log _{10}$ scale.
Extended Data Fig. 7 | Risk of incident post-acute sequelae in people wit COVID-19 who were hospitalized for COVID-19. a-c, Incident diagnoses (a), incident medication use (b) and incident laboratory abnormalities (c). People who had been hospitalized for other causes served as the referent category. 


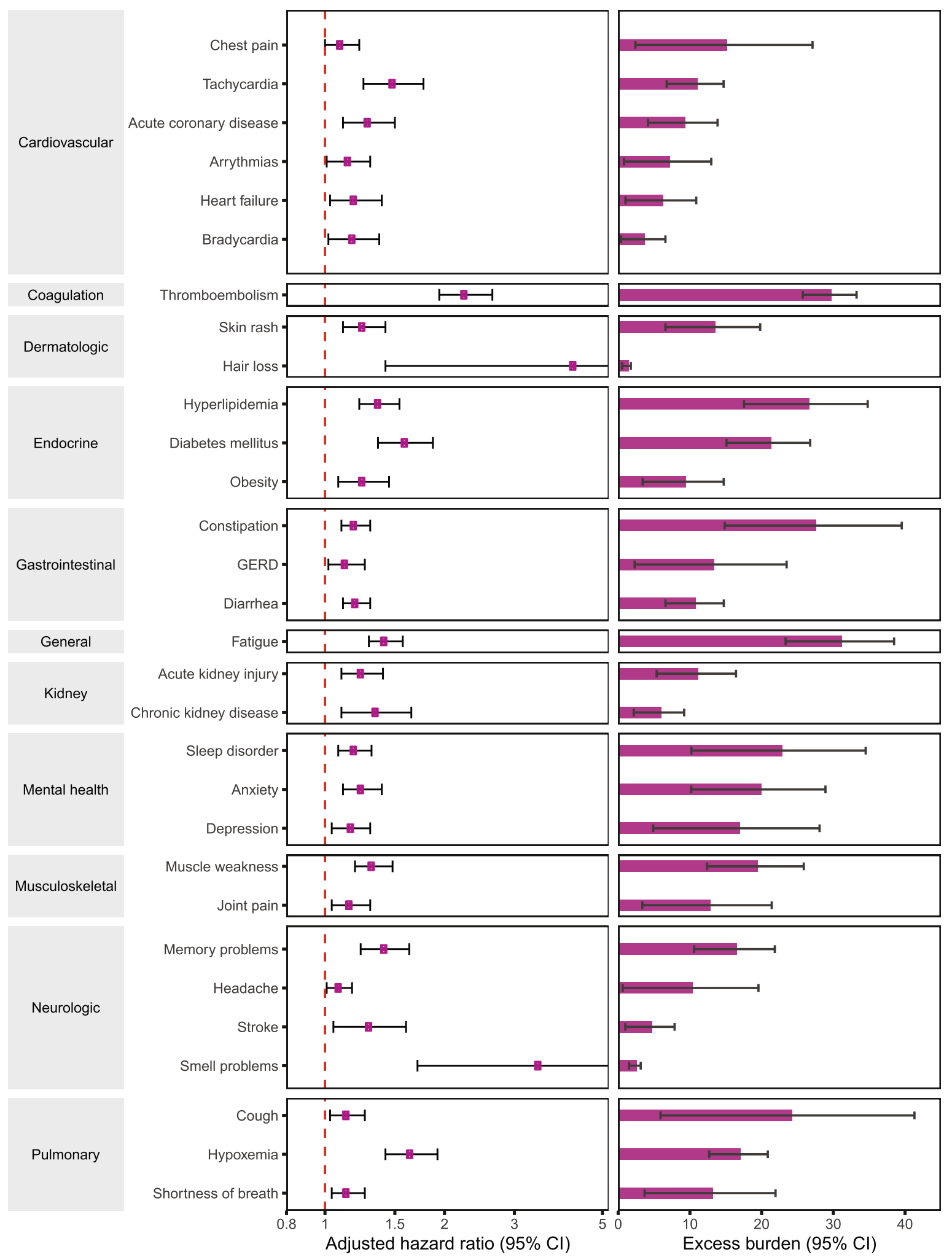

Extended Data Fig. 8 | Risks and burdens of incident prespecified high-resolution post-acute COVID-19 outcomes at 6 months in hospitalized people with COVID-19 versus seasonal inf luenza. Hospitalized people with seasonal influenza served as the referent category. Outcomes were ascertained from day 30 after hospital admission until the end of follow-up. Hazard ratios and $95 \%$ confidence intervals and excess burdens per 1,000 patients and $95 \%$ confidence intervals at 6 months are presented. 


\section{Article}

\section{Extended Data Table 1| Characteristics of study cohorts}

\begin{tabular}{|c|c|c|c|c|}
\hline \multirow[b]{2}{*}{ Characteristics } & \multicolumn{2}{|c|}{ COVID-19 vs. VHA users } & \multicolumn{2}{|c|}{ Hospitalized COVID-19 vs. seasonal Influenza } \\
\hline & $\begin{array}{l}\text { COVID }-19 \\
\mathrm{~N}=73,435\end{array}$ & $\begin{array}{l}\text { VHA users } \\
\mathrm{N}=4,990,835\end{array}$ & $\begin{array}{l}\text { Hospitalized COVID-19 } \\
\qquad N=13,654\end{array}$ & $\begin{array}{l}\text { Hospitalized seasonal } \\
\text { influenza } N=13,997\end{array}$ \\
\hline Age (IQR) & $60.70(47.58,71.59)$ & $66.68(51.87,73.91)$ & $70.25(60.69,75.72)$ & $70.14(62.98,77.04)$ \\
\hline \multicolumn{5}{|l|}{ Race (\%) } \\
\hline White & $51,601(70.27)$ & $3,826,222(76.66)$ & $8120(59.47)$ & $10,235(73.12)$ \\
\hline Black & $18,287(24.90)$ & $930,798(18.65)$ & $4610(33.76)$ & $3124(22.32)$ \\
\hline Other & $3547(4.83)$ & $233,815(4.68)$ & $924(6.77)$ & $638(4.56)$ \\
\hline \multicolumn{5}{|l|}{ Gender (\%) } \\
\hline Male & $64,555(87.91)$ & $4,514,365(90.45)$ & $12,861(94.19)$ & $13,207(94.36)$ \\
\hline Female & $8880(12.09)$ & $476,470(9.55)$ & $793(5.81)$ & $790(5.64)$ \\
\hline Number of outpatient encounter (IQR) * & $3(2,5)$ & $2(1,4)$ & $7(5,11)$ & $9(5,13)$ \\
\hline Number of hospital admission (IQR) * & $0(0,0)$ & $0(0,0)$ & $0(0,1)$ & $0(0,1)$ \\
\hline Number of prescriptions received (IQR) * & $8(4,13)$ & $6(3,11)$ & $12(7,18)$ & $16(8,23)$ \\
\hline Number of outpatient eGFR measurements (IQR) a & $1(1,2)$ & $1(0,2)$ & $4(2,8)$ & $5(2,10)$ \\
\hline Area deprivation index (IQR) & $54.31(43.84,62.99)$ & $53.71(41.89,62.60)$ & $54.05(42.87,61.39)$ & $52.82(40.12 .61 .31)$ \\
\hline Sequential Organ Failure Assessment Score (IQR) & NA & NA & $1(0,2)$ & $1(0,2)$ \\
\hline Follow up days (IQR) & $126(81,203)$ & $130(82,205)$ & $150(84,217)$ & $157(87,220)$ \\
\hline Total Person-years (Sum) & $29,723.73$ & $2,040,891.79$ & 5959.01 & 6220.04 \\
\hline
\end{tabular}

b

\begin{tabular}{|c|c|c|c|c|}
\hline Characteristic & $\begin{array}{l}\text { VHA users } \\
\mathrm{N}=4,990,835\end{array}$ & $\begin{array}{c}\text { CovID-19 without } \\
\text { hospitalization } \\
N=73,435\end{array}$ & $\begin{array}{l}\text { Hospitalized COVID-19 without } \\
\text { admit to intensive care } \\
\qquad N=10,068\end{array}$ & $\begin{array}{l}\text { Hospitalized COVID-19 } \\
\text { admitted to intensive care } \\
N=3586\end{array}$ \\
\hline Age (IQR) & $66.68(51.87,73.91)$ & $60.70(47.58,71.59)$ & $70.07(60.32,75.83)$ & $70.40(61.87,75.53)$ \\
\hline \multicolumn{5}{|l|}{ Race (\%) } \\
\hline White & $3,826,222(76.66)$ & $51,601(70.27)$ & $5969(59.29)$ & $2151(59.98)$ \\
\hline Other & $233,815(4.68)$ & $3547(4.83)$ & $682(6.77)$ & $242(6.75)$ \\
\hline \multicolumn{5}{|l|}{ Gender (\%) } \\
\hline Male & $4,514,365(90.45)$ & $64,555(87.91)$ & $9470(94.06)$ & $3391(94.56)$ \\
\hline Female & $476,470(9.55)$ & $8880(12.09)$ & $598(5.94)$ & $195(5.44)$ \\
\hline Long term care $(\%)$ & $31,944(0.64)$ & $2462(3.35)$ & $1305(12.96)$ & $401(11.18)$ \\
\hline Number of hospital admission (IQR) * & $0(0,0)$ & $0(0,0)$ & $0(0,1)$ & $0(0,1)$ \\
\hline Number of prescriptions received (IQR) * & $6(3,11)$ & $8(4,13)$ & $12(7,18)$ & $12(7,18)$ \\
\hline $\begin{array}{l}\text { Number of outpatient eGFR } \\
\text { measurements (IQR) * }\end{array}$ & $1(0,2)$ & $1(1,2)$ & $4(2,8)$ & $4(2,9)$ \\
\hline Area deprivation index (IQR) & $53.71(41.89,62.60)$ & $54.31(43.84,62.99)$ & $53.69(42.87,61.31)$ & $54.53(42.87,61.99)$ \\
\hline Follow up days (IQR) & $130(82,205)$ & $126(81,203)$ & $151(83,217)$ & $145(85,217)$ \\
\hline Total Person-years (Sum) & $2,040,891.79$ & $29,723.73$ & 4409.01 & 1550.00 \\
\hline
\end{tabular}

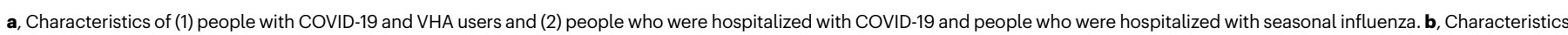

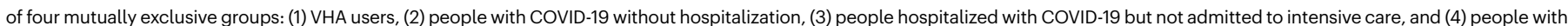
COVID-19 and admitted to intensive care.

*Data collected within one year before the cohort enrolment. 


\section{Extended Data Table 2 | Results of negative controls, and evidence of high risk of death and health resource use}

\begin{tabular}{|c|c|c|}
\hline Negative outcome control & $\begin{array}{c}\text { COVID-19 vs. VHA users } \\
\begin{array}{c}\text { Hazard Ratio } \\
\text { (95\% confidence interval) } *, ~\end{array}\end{array}$ & $\begin{array}{c}\begin{array}{c}\text { Hospitalized COVID-19 vs. } \\
\text { Hospitalized Seasonal Influenza }\end{array} \\
\begin{array}{c}\text { Hazard Ratio } \\
(95 \% \text { confidence interval }) \text { t, } \neq\end{array}\end{array}$ \\
\hline Accidental injuries & $1.03(0.95,1.12)$ & $1.02(0.90,1.15)$ \\
\hline Scars & $0.98(0.67,1.44)$ & $1.06(0.38,2.95)$ \\
\hline Fitting or adjustment of other devices (orthodontic or dental prosthetic device) & $1.04(0.95,1.14)$ & $0.96(0.74,1.24)$ \\
\hline Fitting or adjustment of hearing aids & $1.04(0.94,1.11)$ & $1.02(0.76,1.37)$ \\
\hline Fitting or adjustment of orthotics & $1.13(0.93,1.38)$ & $1.06(0.62,1.82)$ \\
\hline
\end{tabular}

b

\begin{tabular}{|c|c|c|c|c|c|}
\hline Cohort & Outcomes & $\begin{array}{l}\text { Hazard ratio } \\
(95 \% \text { confidence } \\
\text { interval }) \neq\end{array}$ & $\begin{array}{l}\text { Incident rate per } 1000 \text { at } 6 \text { - } \\
\text { months in COVID-19 group } \\
(95 \% \text { confidence interval) } \neq\end{array}$ & $\begin{array}{l}\text { Incident rate per } 1000 \text { at } 6- \\
\text { months in comparison group } \\
\left(95 \% \text { confidence interval) }{ }^{\ddagger}\right.\end{array}$ & $\begin{array}{c}\text { Excess burden per } 1000 \\
\text { at } 6 \text {-months ( } 95 \% \\
\text { confidence interval) } \ddagger\end{array}$ \\
\hline COVID-19 & Death & $1.59(1.46,1.73)$ & $22.77(20.90,24.81)$ & $14.38(13.19,15.68)$ & $8.39(7.09,9.58)$ \\
\hline $\begin{array}{l}\text { vs. } \\
\text { VHA users * }\end{array}$ & $\begin{array}{l}\text { Outpatient } \\
\text { encounter }\end{array}$ & $1.20(1.19,1.21)$ & $946.69(944.94,948.41)$ & $913.48(911.11,915.81)$ & $33.22(30.89,35.58)$ \\
\hline $\begin{array}{l}\text { Hospitalized } \\
\text { COVID-19 }\end{array}$ & Death & $1.51(1.30,1.76)$ & $87.92(76.11,101.47)$ & $59.13(51.07,68.41)$ & $28.79(19.52,36.85)$ \\
\hline $\begin{array}{l}\text { VS. } \\
\text { hospitalized } \\
\text { seasonal } \\
\text { influenza }{ }^{\dagger}\end{array}$ & $\begin{array}{l}\text { Outpatient } \\
\text { encounter }\end{array}$ & $1.12(1.08,1.17)$ & $990.32(988.50,991.90)$ & $983.95(981.28,986.30)$ & $6.37(4.01,9.03)$ \\
\hline
\end{tabular}

C

\begin{tabular}{|c|c|c|c|}
\hline \multirow[b]{2}{*}{ Cohort } & \multicolumn{3}{|c|}{ Number of outpatient encounters per 30 days } \\
\hline & $\begin{array}{l}\text { In COVID-19 group } \\
(95 \% \text { confidence interval) }\end{array}$ & $\begin{array}{c}\text { In comparison group (VHA users or } \\
\text { seasonal influenza) } \\
(95 \% \text { confidence interval }) \ddagger\end{array}$ & $\begin{array}{c}\text { Excess encounters } \\
(95 \% \text { confidence interval) }\end{array}$ \\
\hline COVID-19 vs. VHA users * & $3.03(3.00,3.05)$ & $2.56(2.56,2.57)$ & $0.47(0.44,0.49)$ \\
\hline $\begin{array}{l}\text { Hospitalized COVID-19 vs. } \\
\text { hospitalized seasonal influenza }\end{array}$ & $7.51(7.37,7.65)$ & $6.06(5.95,6.16)$ & $1.45(1.28,1.63)$ \\
\hline
\end{tabular}

a, Results of testing negative outcome controls in people with COVID-19 compared to VHA users, and in people who had been hospitalized with COVID-19 compared to people who had

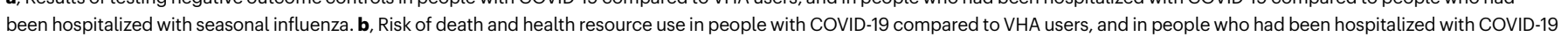

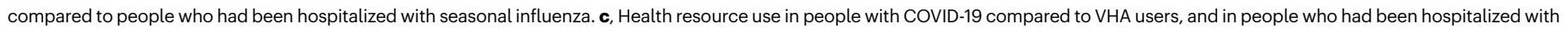
COVID-19 compared to people who had been hospitalized with seasonal influenza.

*For analyses of people with COVID-19 versus VHA users, outcomes were ascertained from 30 days after COVID-19 diagnosis and VHA users served as the referent category.

†For analyses of people who had been hospitalized with COVID-19 versus people who had been hospitalized with seasonal influenza, outcomes were ascertained from 30 days after hospital admission and people who had been hospitalized with seasonal influenza served as the referent category.

₹Results based on models adjusted through overlap weighting. 


\section{Reporting Summary}

Nature Research wishes to improve the reproducibility of the work that we publish. This form provides structure for consistency and transparency in reporting. For further information on Nature Research policies, see our Editorial Policies and the Editorial Policy Checklist.

\section{Statistics}

For all statistical analyses, confirm that the following items are present in the figure legend, table legend, main text, or Methods section.

n/a $\mid$ Confirmed

$\bigotimes$ The exact sample size $(n)$ for each experimental group/condition, given as a discrete number and unit of measurement

$\bigotimes$ A statement on whether measurements were taken from distinct samples or whether the same sample was measured repeatedly

The statistical test(s) used AND whether they are one- or two-sided

Only common tests should be described solely by name; describe more complex techniques in the Methods section.

$\bigotimes$ A description of all covariates tested

$\bigotimes$ A description of any assumptions or corrections, such as tests of normality and adjustment for multiple comparisons

$\triangle$ A full description of the statistical parameters including central tendency (e.g. means) or other basic estimates (e.g. regression coefficient)

AND variation (e.g. standard deviation) or associated estimates of uncertainty (e.g. confidence intervals)

$\varnothing$ For null hypothesis testing, the test statistic (e.g. $F, t, r$ ) with confidence intervals, effect sizes, degrees of freedom and $P$ value noted Give $P$ values as exact values whenever suitable.

Х $\square$ For Bayesian analysis, information on the choice of priors and Markov chain Monte Carlo settings

Х $\square$ For hierarchical and complex designs, identification of the appropriate level for tests and full reporting of outcomes

Х $\square$ Estimates of effect sizes (e.g. Cohen's d, Pearson's $r$ ), indicating how they were calculated

Our web collection on statistics for biologists contains articles on many of the points above.

\section{Software and code}

Policy information about availability of computer code

Data collection SAS Enterprise Guide version 7.1 was used to collect data for the study. ICD-10 diagnosis codes were classified into 540 diagnostic categories based on the Clinical Classifications Software Refined (CCSR) version 2021.1 (Healthcare Cost and Utilization Project, Agency for Healthcare Research and Quality).

Data analysis All analyses were done using SAS Enterprise Guide version 7.1 (SAS Institute, Cary, NC). Data visualizations were performed in R 4.0.3 (R Foundation for Statistical Computing, Vienna, Austria).

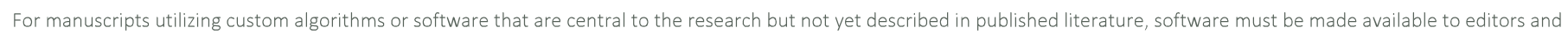

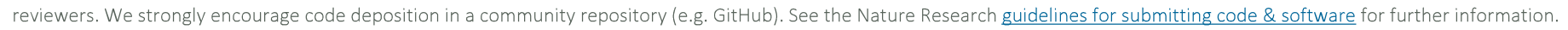

\section{Data}

Policy information about availability of data

All manuscripts must include a data availability statement. This statement should provide the following information, where applicable:

- Accession codes, unique identifiers, or web links for publicly available datasets

- A list of figures that have associated raw data

- A description of any restrictions on data availability

The data are available from the US Department of Veterans Affairs 


\section{Field-specific reporting}

Please select the one below that is the best fit for your research. If you are not sure, read the appropriate sections before making your selection.

\ Life sciences $\quad \square$ Behavioural \& social sciences $\quad \square$ Ecological, evolutionary \& environmental sciences

For a reference copy of the document with all sections, see nature.com/documents/nr-reporting-summary-flat.pdf

\section{Life sciences study design}

All studies must disclose on these points even when the disclosure is negative.

Sample size To achieve better precision of the study results, we enrolled all users of the US Veterans Health Administration and followed them until January 31, 2021. To our knowledge, this is the largest and most comprehensive post-acute COVID-19 study to date involving 73,435 nonhospitalized patients with COVID-19, and 4,990,835 controls (2,070,615.52 person years of follow-up), and 13,654 hospitalized patients with COVID-19, and 13,997 patients hospitalized with seasonal influenza (12,179.05 person years of follow-up).

Data exclusions To examine the risk of post-acute outcomes beyond the first 30 days of illness, we predefined our exclusion criteria and excluded participants who did not survive the first 30 days of COVID-19 illness.

Replication To provide results with better accuracy and precision, we included all users of the US Veterans Health Administration to estimate the study results. The finding was not replicated because no other internal or external datasets with large sample size and high dimensional information is currently available to us.

Randomization Exposure allocation was not random. To adjust for potential confounders, we applied overlap weighting based on propensity scores constructed from a) predefined variables including demographic, healthcare utilization and contextual factors, and b) from algorithmically selected high dimensional variables from domains including diagnoses, pharmacy records, and laboratory tests.

\section{Reporting for specific materials, systems and methods}

We require information from authors about some types of materials, experimental systems and methods used in many studies. Here, indicate whether each material, system or method listed is relevant to your study. If you are not sure if a list item applies to your research, read the appropriate section before selecting a response.

Materials \& experimental systems

$\mathrm{n} / \mathrm{a}$ Involved in the study

\ $\square$ Antibodies

Х $\square$ Eukaryotic cell lines

\ $\square$ Palaeontology and archaeology

\begin{tabular}{l|l} 
Methods \\
n/a & Involved in the study
\end{tabular}

Х

Animals and other organisms

$\square$ \uman research participants

\ $\square$ Clinical data

$\bigotimes \square$ Dual use research of concern

\section{Human research participants}

\section{Policy information about studies involving human research participants}

Population characteristics

Study participants are users of the US Veteran Health Administration. The overall study population had a median age of 67 $87 \%$ were male, and $77 \%$ of cohort participants were of white race. 73,435 people with COVID-19 and 4,990,835 controls were evaluated. Additionally, 13,654 people hospitalized with COVID-19 and 13,997 people hospitalized with seasonal influenza were also evaluated. The hospitalized group comprised of 10,068 patients who were hospitalized but did not require intensive care, and 3,586 patients who were admitted to intensive care.

Recruitment

Participants were recruited if they had at least 1 encounter with the US Veteran Health Administration in the year prior to cohort enrollment. Non users of the VA health care system were not included. The characteristics of the study population may be different from the general population (US or global population). Other biases due to recruitment including selfselection bias are unlikely to bias the results of this study.

Ethics oversight 